\title{
SÍNTESE DE 2-(2-PIRIDIL)QUINOLINAS PROMOVIDA POR MICRO-ONDAS E SUAS ATIVIDADES ANTIFÚNGICAS
}

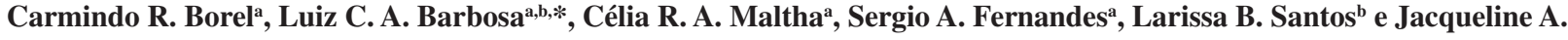 \\ Takahashi $^{\mathrm{b}}$ \\ aDepartamento de Química, Universidade Federal de Viçosa, Av. P. H. Rolfs s/n, 36570-900 Viçosa, MG, Brasil. \\ ${ }^{\text {b} D e p a r t a m e n t o ~ d e ~ Q u i ́ m i c a, ~ I n s t i t u t o ~ d e ~ C i e ̂ n c i a s ~ E x a t a s, ~ U n i v e r s i d a d e ~ F e d e r a l ~ d e ~ M i n a s ~ G e r a i s, ~ A v . ~ P r e s . ~ A n t o ̂ n i o ~ C a r l o s, ~ 6627, ~}$ \\ Campus Pampulha, CEP 31270-901, Belo Horizonte, MG, Brasil.
}

Recebido em 01/06/2017; aceito em 27/07/2017; publicado na web em 12/09/2017

\begin{abstract}
SYNTHESIS OF 2-(2-PYRIDYL)QUINOLINES PROMOTED BY MICROWAVES AND THEIR ANTIFUNGAL ACTIVITIES. In this work a series of 2-(2- pyridyl)quinolines were prepared via a Povarov reaction between anilines, 2-pyridinocarbadehyde and ethyl vinyl ether under microwaves heating conditions. The optimized conditions herein reported allowed the preparation of several pyridylquinolines in yields in the range of $30-83 \%$, some of them not previously accessible by this multicomponent process. The reported methodology has advantage over previous report due to its larger scope and short reaction time (2 hours). All quinolines obtained were assayed against five species of clinically important yeasts Candida sp and against Cryptococcus neoformans. Some of them possessed a broad spectrum of action including 2-(2-pyridyl)quinoline (20) and 6,8-dimethoxy-2-(pyridin-2-yl)quinolone (22) that were highly effective in inhibiting Candida species $\left(\mathrm{IC}_{50}<1.95 \mu \mathrm{g} / \mathrm{mL}\right.$ against $C$. tropicalis and $C$. krusei). Some compounds were more potent than commercial drugs Nistatin and Miconazole.
\end{abstract}

Keywords: microwave; Povarov multicomponent reactions; pyridylquinoline; antifungal activity.

\section{INTRODUÇÃO}

A incidência de infecções fúngicas oportunistas, causadas por patógenos endógenos como leveduras do gênero Candida ou adquiridos no meio ambiente (Aspergillus, Cryptococcus), ${ }^{1-3} \mathrm{vem}$ aumentando dramaticamente nas últimas décadas. ${ }^{4}$ As taxas de morbidade e mortalidade associadas às infecções fúngicas são bastante expressivas, tornando-as claramente um problema persistente de saúde pública.,

Infecções invasivas causadas por Candida spp., Cryptococcus neoformans, Aspergillus spp., Pneumocystis carinii e Histoplasma capsulatum são ameaças constantes à saúde humana. ${ }^{1,2}$ Infecções causadas por fungos do gênero Aspergillus e leveduras da família Candida spp. tem incidência mundial. ${ }^{1,57-9}$ Das espécies de Candida de interesse clínico, C. albicans é o patógeno mais comum e possui elevada virulência. Trata-se de um fungo que habita as cavidades oral e vaginal e o sistema digestivo do homem, sem que isso implique em efeitos prejudiciais à sua saúde. O crescimento desordenado de C. albicans, estado patológico denominado candidíase, surge em decorrência de desequilíbrio na flora local devido ao uso de antibióticos ou a alterações no sistema imunológico em decorrência do acometimento por outras doenças como o diabetes. ${ }^{1} \mathrm{O}$ desafio atual no estudo de doenças infectocontagiosas envolve a busca de novas classes de fármacos, com novos mecanismos de ação e que sejam mais eficazes e menos tóxicos ao homem. ${ }^{4,10}$

Plantas, micro-organismos terrestres e marinhos são fontes potencialmente promissoras de novos compostos bioativos diversos, ${ }^{11-13}$ incluindo agentes antifúngicos. ${ }^{14,15}$

Muitos dos compostos bioativos produzidos por micro-organismos são heterocíclicos. ${ }^{16}$ Dentre os heterociclos nitrogenados, quinolinas e derivados são de grande interesse, uma vez que diversos deles apresentam atividades antiparasitária, ${ }^{17}$ antibacteriana, ${ }^{18}$ antifúngica, ${ }^{1}$ antitumoral, ${ }^{19}$ anti-inflamatória, ${ }^{20}$ antimalárica, ${ }^{21}$ e anti-HIV. ${ }^{22}$

*e-mail: lcab@ufmg.br
Exemplos de quinolinas naturais bioativas incluem a estreptonigrina e lavendamicina (Figura 1), metabólitos fúngicos isolados de Streptomyces flocculus e Streptomyces lavendulae, respectivamente, com propriedades antitumorais, citotóxica e antibacteriana. ${ }^{23-26} \mathrm{Em}$ função dessas atividades, diversas metodologias sintéticas têm sido desenvolvidas para a obtenção desses compostos e seus análogos para fins de estudos de suas propriedades farmacológicas. ${ }^{27-29}$ Notadamente, tem sido descrita a atividade antifúngica de quinolinas substituídas com estrutura geral 3 (Figura 1) contra diversas espécies de Candida, ${ }^{10}$ bem como o potencial antimicrobiano de compostos 4 contra C. albicans, Rhodotorulla bogoriensis e Aspergilus flavus. ${ }^{30}$

Em função das importantes atividades biológicas de diversas quinolinas, relatamos recentemente uma metodologia de síntese para compostos com a unidade 2-(2-piridil)quinolina (5-8) empregando a reação multicomponente de Povarov. ${ }^{31}$ A reação de Povarov é uma metodologia conveniente se comparada à síntese clássica para quinolinas em termos de eficiência, rapidez e economia de átomos. A reação de Povarov multicomponentes é uma aza-Diels-Alder de demanda inversa de elétrons entre uma anilina, um aldeído e um alquino ou alqueno ricos em densidade eletrônica para, em uma única etapa, dar acesso a compostos das classes como tetraidroquinolina, quinolina ou julolidinas.

Nesse trabalho descrevemos a síntese de uma série de 2-(2-piridil) quinolinas, a exemplo dos compostos 5-8 $\mathbf{8}^{31}$ (Figura 1), partindo-se de anilinas substituídas, 2-piridinocarbaldeido e etil vinil éter. Nas condições otimizadas anteriormente relatadas, ${ }^{31}$ a reação foi realizada sob aquecimento a $82{ }^{\circ} \mathrm{C}$ e utilizando $\mathrm{BF}_{3} \cdot \mathrm{O}\left(\mathrm{CH}_{3}\right)_{2}$ como catalisador. Embora bons rendimentos tenham sido obtidos para diversos compostos, em alguns casos a metodologia se mostrou ineficiente, não sendo possível obter os produtos desejados partindo de anilinas contendo grupos retiradores de elétrons, como halogênios. Apesar dessa limitação, o método desenvolvido constituiu-se no mais geral para a síntese desse tipo de composto, uma vez que encontramos apenas um exemplo da aplicação da reação de Povarov para a obtenção de 2-(2-piridil)quinolina, e com um modesto rendimento de $42 \% .^{10}$ 
<smiles>COC1=C(N)C(=O)c2nc(-c3nc(C(=O)O)c(C)c(-c4ccc(OC)c(OC)c4O)c3N)ccc2C1=O</smiles>

$1( \pm)$-Estreptonigrina<smiles></smiles>

2 Lavendamicina<smiles>[R2]c1cc([R])c2ccc(-c3ccccc3)nc2c1</smiles>

$3 \mathrm{R}_{1}, \mathrm{R}_{2}=$ Alquil, Halogênio ou $\mathrm{H}$<smiles>[R]c1nc2ccccc2cc1NCCCCO</smiles>

$4 \mathrm{R}=\mathrm{H}$ ou $\mathrm{CH}_{3}$<smiles>[R]c1c([R])c([R])c2nc(-c3cccc(Br)n3)ccc2c1[R]</smiles>

$5 \mathrm{R}_{1}=\mathrm{R}_{2}=\mathrm{R}_{3}=\mathrm{OCH}_{3} ; \mathrm{R}_{4}=\mathrm{H}$

$6 \mathrm{R}_{2}=\mathrm{R}_{3}=\mathrm{OCH}_{3} ; \mathrm{R}_{1}=\mathrm{R}_{4}=\mathrm{H}$

$7 \mathrm{R}_{4}=\mathrm{OCH}_{3} ; \mathrm{R}_{1}=\mathrm{R}_{2}=\mathrm{R}_{3}=\mathrm{H}$

$8 \mathrm{R}_{2}=\mathrm{OCH}_{3} ; \mathrm{R}_{1}=\mathrm{R}_{3}=\mathrm{R}_{4}=\mathrm{H}$

Figura 1. Estruturas de quinolinas bioativas naturais 1-2 e sintéticas 3-8.

Dando continuidade aos nossos estudos na área de síntese de compostos heterocíclicos com atividade biológica, ${ }^{32-34}$ nesse artigo apresentamos os resultados relativos ao uso de radiação micro-ondas como fonte de aquecimento para a reação de Povarov na síntese de piridinoquinolinas. Também são apresentados os resultados dos ensaios de avaliação antifúngica contra cepas de Candida spp. e Cryptococcus neoformans.

\section{PARTE EXPERIMENTAL}

\section{Procedimentos gerais e equipamentos}

Os reagentes utilizados nas reações e os solventes empregados em cromatografia em coluna de sílica gel foram previamente purificados e a água residual removida conforme os procedimentos descritos na literatura. ${ }^{35}$ Todas as reações foram monitoradas por cromatografia em camada delgada utilizando-se placas de sílica gel Camlab-Polygram $\mathrm{SILK} / \mathrm{UV}_{254}$, com $0,25 \mathrm{~mm}$ de espessura. As placas foram visualizadas sob luz ultravioleta $(254 \mathrm{~nm})$ ou reveladas com solução de ácido fosfomolíbdico. Para a purificação dos compostos utilizou-se a cromatografia em coluna de sílica gel 60 (230-400 mesh-ASTM, Merck). As reações assistidas por micro-ondas foram realizadas em reator DISCOVER SYSTEM (CEM), utilizando acetonitrila como solvente, e com monitorização contínua de temperatura $\left(95^{\circ} \mathrm{C}\right)$ e potência $(50 \mathrm{~W})$ por 2 horas.

As temperaturas de fusão, não corrigidas, foram determinadas em aparelho MQAPF-301. Os espectros no infravermelho (IV) foram obtidos em espectrofotômetro FT-IR VARIAN 660 equipado com GladiATR, sendo realizados por reflectância total atenuada.

Os espectros de $\mathrm{RMN}$ de ${ }^{1} \mathrm{H}$ foram adquiridos em um espectrômetro Varian Mercury-300 e os espectros foram processados usando o software Varian. A frequência operacional foi $300,069 \mathrm{MHz}$ para ${ }^{1} \mathrm{H}$ e 75,459 para ${ }^{13} \mathrm{C}$ com sonda de $5 \mathrm{~mm}$ e deteç̧ão direta e unidade de controle de temperatura, sendo os deslocamentos químicos $(\delta)$ relativos ao tetrametilsilano (TMS, $\delta=0,0$ ), com as amostras dissolvidas em $\mathrm{CDCl}_{3}$. Os espectros de massas de baixa resolução foram obtidos em espectrômetro Shimadzu QP5050A. O aparelho foi equipado com uma coluna SE54 (30 $\mathrm{m}$ x 0,25 $\mathrm{mm} \times 0,25 \mu \mathrm{m}$ ), utilizando Hélio como gás de arraste, com fluxo de $1,6 \mathrm{~mL} \mathrm{~min}^{-1}$. O injetor e o detector de ionização de chamas foram mantidos a $290^{\circ} \mathrm{C}$. O forno foi programado para permanecer a $40{ }^{\circ} \mathrm{C}$ por 2 minutos, sendo então aquecido na taxa de $20^{\circ} \mathrm{C}$ por minuto até $300^{\circ} \mathrm{C}$, permanecendo nesta temperatura por 10 minutos. Os espectros de massas de alta resolução no modo de ionização por electrospray foram registrados em equipamento Finnigan MAT LCQ 7000.

\section{Procedimentos sintéticos}

5,6,8-trimetoxi-2-(piridin-2-il)quinolina 12. Em um frasco de vidro de $10 \mathrm{~mL}$, próprio para forno de micro-ondas, foram adicionados 3,4,5-trimetoxianilina (183 mg; 1,0 mmol), 2-piridinocarbaldeído (214 $\mathrm{mg} ; 2,0 \mathrm{mmol})$, etil vinil éter (216 mg; 3,00 mmol), $\mathrm{BF} 3 . \mathrm{O}\left(\mathrm{CH}_{3}\right)_{2}(46$ $\mu \mathrm{L} ; 0,6 \mathrm{mmol} ; 30 \mathrm{~mol} \%)$ e acetonitrila anidra $(4 \mathrm{~mL})$. O tubo foi selado e colocado em um aparelho micro-ondas modelo CEM Discover. O método utilizado no aparelho de micro-ondas para o experimento foi o Padrão (Standard), utilizando ar comprimido em todas as análises. A mistura foi pré-agitada durante 5 min (tempo de rampa), irradiada na potência de $50 \mathrm{~W}$, a $95{ }^{\circ} \mathrm{C}$ durante 120 minutos. A mistura reacional foi então transferida para um frasco Erlenmeyer contendo uma solução saturada de bicarbonato de sódio $(10 \mathrm{~mL})$. O sólido foi removido por filtração e a mistura aquosa foi extraída com acetato de etila ( 3 x 15 $\mathrm{mL}$ ). A fase orgânica foi tratada com sulfato de magnésio anidro, filtrada e concentrada sob pressão reduzida em evaporador rotativo resultando em um sólido amarelo. O sólido foi purificado por cromatografia em coluna de sílica gel eluída com mistura de hexano/acetato de etila (3:1 $\mathrm{v} / \mathrm{v})$, resultando no produto desejado (237 mg; 0,8 mmol) em $80 \%$ de rendimento. O composto foi finalmente recristalizado em hexano/ diclorometano, fornecendo um sólido branco.

Os compostos 12-26 foram sintetizados utilizando o mesmo procedimento experimental descrito para o composto $\mathbf{1 2}$.

\section{Dados físicos e espectroscópicos}

5,6,8-trimetoxi-2-(piridin-2-il)quinolina 12: sólido branco $(0,80$ mmol, 80\%), Tf $=147-148^{\circ} \mathrm{C} . \mathrm{IV}\left(\mathrm{cm}^{-1}\right) \overline{\mathrm{v}}_{\max }: 3054 ; 2936 ; 2830 ; 2362$; $1615 ; 1590 ; 1477 ; 1398 ; 1235 ; 1100 ; 993 ; 802 ; 655 ; 619$. RMN de ${ }^{1} \mathrm{H}\left(300 \mathrm{MHz}, \mathrm{CDCl}_{3}\right) \delta 4,00\left(\mathrm{~s}, 3 \mathrm{H}, \mathrm{OCH}_{3}\right) ; 4,04$ (s, 3H, $\left.\mathrm{OCH}_{3}\right)$; 
4,08 (s, 3H, $\left.\mathrm{OCH}_{3}\right) ; 7,34$ (ddd, $1 \mathrm{H}, J=7,9 \mathrm{~Hz}, J=4,8 \mathrm{~Hz}$ e $J=1,1$ Hz, H-5'); 7,37 (sl, 1H, H-8); 7,85 (dt, $1 \mathrm{H}, J=7,9 \mathrm{~Hz}$ e $J=1,4 \mathrm{~Hz}$, H-4'); 8,39 (d, $1 \mathrm{H}, J=8,7 \mathrm{~Hz}, \mathrm{H}-3$ ); 8,48 (dd, $1 \mathrm{H}, J=8,7 \mathrm{~Hz}$ e $J$ $=0,5 \mathrm{~Hz}, \mathrm{H}-4) ; 8,57$ (ddd, $1 \mathrm{H}, J=7,9 \mathrm{~Hz}, J=1,1 \mathrm{~Hz}$ e $J=0,8 \mathrm{~Hz}$, H-3'); 8,73 (ddd, $1 \mathrm{H}, J=4,8 \mathrm{~Hz}, J=1,4 \mathrm{~Hz}$ e $J=0,8 \mathrm{~Hz}, \mathrm{H}-6$ '). $\mathrm{RMN}$ de ${ }^{13} \mathrm{C}\left(75 \mathrm{MHz}, \mathrm{CDCl}_{3}\right) \delta 56,4\left(\mathrm{OCH}_{3}\right) ; 61,5\left(\mathrm{OCH}_{3}\right) ; 61,9$ $\left(\mathrm{OCH}_{3}\right) ; 104,4$ (C-8); 116,9 (C-5'); 119,6 (C-9); 122,1 (C-3'); 124,2 (C-3); 131,8 (C-4); 137,2 (C-4'); 141,4 (C-6); 145,5 (C-10); 147,1 (C-5); 149,5 (C-6'); 155,5 (C-2); 156,2 (C-7); 156,5 (C-2'). EM, m/z (\%) $296\left(\mathrm{C}_{17} \mathrm{H}_{16} \mathrm{~N}_{2} \mathrm{O}_{3}\right)\left(\left[\mathrm{M}^{+}\right], 100\right) ; 297\left([\mathrm{M}+1]^{+}, 19\right) ; 282$ (9); 281 (50); 254 (8); 253 (49); 239 (5); 238 (40); 223 (17); 210 (14); 209 (6); 179 (5); 167 (32); 166 (12); 156 (6); 148 (8); 140 (5); 139 (14); 133 (7); 119 (10); 111 (7); 97 (5); 90 (5); 89 (6); 83 (10); 78 (12); 70 (5); 63 (10); 62 (5); 51 (7). HRMS [ESI(+),IT-TOF]: calculado $\left[\mathrm{C}_{17} \mathrm{H}_{17} \mathrm{~N}_{2} \mathrm{O}_{3}\right]^{+}$297,1234; encontrado 297,1093.

5,8-dimetoxi-2-(piridin-2-il)quinolina 13: sólido amarelo $(0,79$ mmol, 79\%), Tf $=133-134^{\circ} \mathrm{C} . \mathrm{IV}\left(\mathrm{cm}^{-1}\right) \overline{\mathrm{v}}_{\text {max }}: 3057,2925,2850,1589$, $1460,1258,1101,781,719,619$. RMN de ${ }^{1} \mathrm{H}\left(300 \mathrm{MHz}, \mathrm{CDCl}_{3}\right)$ $\delta 3,89\left(\mathrm{~s}, 3 \mathrm{H}, \mathrm{OCH}_{3}\right) ; 4,08\left(\mathrm{~s}, 3 \mathrm{H}, \mathrm{OCH}_{3}\right) ; 6,78(\mathrm{~d}, 1 \mathrm{H}, J=8,5 \mathrm{~Hz}$, H-6); 6,98 (d, 1H, $J=8,5 \mathrm{~Hz}, \mathrm{H}-7) ; 7,34$ (ddd, $1 \mathrm{H}, J=7,8 \mathrm{~Hz}, J=$ $4,8 \mathrm{~Hz}$ e $J=1,0 \mathrm{~Hz}, \mathrm{H}-5$ '); 7,86 (dt, $1 \mathrm{H}, J=7,8 \mathrm{~Hz}$ e $J=1,4 \mathrm{~Hz}$, H-4'); 8,58 (d, 1H, $J=8,8 \mathrm{~Hz}, \mathrm{H}-3$ ); 8,68 (d, 1H, $J=8,8 \mathrm{~Hz}, \mathrm{H}-4)$; $8,72-8,75$ (m, 2H, H-3' e H-6'). RMN de ${ }^{13} \mathrm{C}\left(75 \mathrm{MHz}, \mathrm{CDCl}_{3}\right) \delta 56,0$ (C-11); 56,6 (C-12); 104,3 (C-6); 107,7 (C-7); 118,8 (C-3); 121,8 (C-9); 122,4 (C-3'); 124,2 (C-5'); 132,1 (C-4); 137,2 (C-4'); 140,4 (C-5); 149,1 (C-10); 149,3 (C-6'); 149,8 (C-8); 155,6 (C-2); 156,5 (C-2'). EM, $m / z$ (\%) $266\left(\mathrm{C}_{16} \mathrm{H}_{14} \mathrm{~N}_{2} \mathrm{O}_{2}\right)\left(\left[\mathrm{M}^{+}\right], 41\right) ; 267$ ([M+1] $\left.]^{+}, 8\right)$; 265 (46); 252 (14); 251 (100); 250 (5); 249 (8); 237 (22); 236 (10); 223 (8); 222 (14); 221 (10); 208 (6); 195 (6); 194 (12); 193 (21); 192 (10); 180 (7); 179 (12); 153 (6); 118 (5); 117 (7); 110 (5); 104 (7); 103 (8); 97 (12); 90 (5); 83 (10); 79 (5); 78 (13); 77 (8); 76 (9); 75 (6); 63 (8); 52 (6); 51 (13). HRMS [ESI(+),IT-TOF]: calculado $\left[\mathrm{C}_{16} \mathrm{H}_{15} \mathrm{~N}_{2} \mathrm{O}_{2}\right]^{+}$267,1128; encontrado 267,1029.

2-(6-bromopiridin-2-il)-5,8-dimetoxiquinolina 14: sólido amarelo (0,61 mmol, 61\%), Tf = 167-168 ${ }^{\circ} \mathrm{C} . \mathrm{IV}\left(\mathrm{cm}^{-1}\right) \overline{\mathrm{v}}_{\text {max }}: 2999,2937,2831$, $1582,1474,1428,1261,1128,1100,986,789$. RMN de ${ }^{1} \mathrm{H}(300 \mathrm{MHz}$, $\left.\mathrm{CDCl}_{3}\right) \delta 3,98$ (s, 3H, H-11); 4,08 (s, 3H, H-12); 6,78 (d, 1H, $J=8,5$ Hz, H-6); 6,97 (d, 1H, $J=8,5 \mathrm{~Hz}, \mathrm{H}-7) ; 7,52$ (dd, $1 \mathrm{H}, J=7,8 \mathrm{~Hz}$ e $J$ $=0,9 \mathrm{~Hz}, \mathrm{H}-5$ ') 7,71 (t, 1H, $J=7,8 \mathrm{~Hz}, \mathrm{H}-4^{\prime}$ ); 8,58 (d, 1H, $J=8,8$ $\mathrm{Hz}, \mathrm{H}-3$ ); 8,68 (d, 1H, $J=8,8 \mathrm{~Hz}, \mathrm{H}-4), 8,70$ (dd, $1 \mathrm{H}, J=7,8 \mathrm{Hze} J$ $\left.=0,9 \mathrm{~Hz}, \mathrm{H}-3{ }^{\prime}\right)$. RMN de ${ }^{13} \mathrm{C}\left(75 \mathrm{MHz}, \mathrm{CDCl}_{3}\right) \delta 56,0(\mathrm{C}-11) ; 56,6$ (C-12); 104,6 (C-6); 107,9 (C-7); 118,9 (C-3); 121,2 (C-3'); 122,1 (C-9); 128,6 (C-5'); 132,4 (C-4); 139,5 (C-4'); 140,1 (C-5); 141,7 (C-6'); 149,0 (C-10); 149,6 (C-8); 153,8 (C-2); 157,4 (C-2'). EM, $\mathrm{m} / \mathrm{z}(\%) 344\left(\mathrm{C}_{16} \mathrm{H}_{13} \mathrm{~N}_{2} \mathrm{O}_{2} \mathrm{Br}\right)\left(\left[\mathrm{M}^{+} \cdot\right], 33\right) ; 346\left([\mathrm{M}+2]^{+}, 31\right) ; 332(13)$; 331 (94); 330 (21); 329 (100); 328 (7); 317 (19); 315 (21); 300 (10); 273 (8); 235 (36); 220 (9); 208 (9); 207 (27); 193 (8); 192 (17); 179 (13); 153 (11); 125 (26); 103 (8); 102 (14); 96 (9); 82 (10); 77 (8); 76 (15); 75 (14); 63 (12); 51 (11); 50 (14). HRMS [ESI(+),IT-TOF]: calculado $\left[\mathrm{C}_{16} \mathrm{H}_{14} \mathrm{~N}_{2} \mathrm{O}_{2} \mathrm{Br}\right]^{+}$345,0233; encontrado 345,0126.

6,7-dimetoxi-2-(piridin-2-il)quinolina 15: sólido amarelo (0,76 mmol, 76\%), Tf $=163-164{ }^{\circ} \mathrm{C} . \mathrm{IV}\left(\mathrm{cm}^{-1}\right) \overline{\mathrm{v}}_{\max }: 3074,2923,2851$, $1620,1457,1233,1157,1000,855,767,622$. RMN de ${ }^{1} \mathrm{H}(300 \mathrm{MHz}$, $\left.\mathrm{CDCl}_{3}\right) \delta 4,04\left(\mathrm{~s}, 3 \mathrm{H}, \mathrm{OCH}_{3}\right) ; 4,08\left(\mathrm{~s}, 3 \mathrm{H}, \mathrm{OCH}_{3}\right) ; 7,09$ (s, 1H, H-5); 7,33 (ddd, $1 \mathrm{H}, J=7,5 \mathrm{~Hz}, J=4,8 \mathrm{~Hz}$ e $J=1,2 \mathrm{~Hz}, \mathrm{H}-5$ '); 7,58 (s, $1 \mathrm{H}$, H-8); 7,86 (dt, $1 \mathrm{H}, J=7,5 \mathrm{~Hz}$ e $J=1,7 \mathrm{~Hz}, \mathrm{H}_{-} 4^{\prime}$ ); 8, 14 (d, $1 \mathrm{H}, J=8,5$ $\mathrm{Hz}, \mathrm{H}-4) ; 8,40$ (d, $1 \mathrm{H}, J=8,5 \mathrm{~Hz}, \mathrm{H}-3$ ); 8,59 (ddd, $1 \mathrm{H}, J=7,5 \mathrm{~Hz}, J$ $=1,2 \mathrm{~Hz}$ e $J=0,9 \mathrm{~Hz}, \mathrm{H}-3$ ') ; 8,73 (ddd, $1 \mathrm{H}, J=4,8 \mathrm{~Hz}, J=1,7 \mathrm{~Hz}$ e $J=0,9 \mathrm{~Hz}, \mathrm{H}-6$ ' $)$. RMN de ${ }^{13} \mathrm{C}\left(75 \mathrm{MHz}, \mathrm{CDCl}_{3}\right) \delta 56,3\left(\mathrm{OCH}_{3}\right) ; 56,4$ $\left(\mathrm{OCH}_{3}\right) ; 105,2$ (C-5); 108,6 (C-8); 117,6 (C-3); 121,6 (C-3'); 123,8
(C-5'); 124,2 (C-9); 135,2 (C-4); 137,1 (C-4'); 145,1 (C-10); 149,4 (C-6'); 150,3 (C-6); 152,7 (C-7); 154,4 (C-2); 156,9 (C-2'). EM, m/z (\%) $266\left(\mathrm{C}_{16} \mathrm{H}_{14} \mathrm{~N}_{2} \mathrm{O}_{2}\right)\left(\left[\mathrm{M}^{+}\right], 100\right) ; 267\left([\mathrm{M}+1]^{+}, 19\right) ; 251(20) ; 224$ (7); 223 (43); 221 (7); 208 (7); 195 (10); 194 (5); 193 (7); 192 (8); 180 (15); 179 (20); 133 (8); $117(5) ; 111(8) ; 102(6) ; 96(5) ; 90(5)$; 83 (5); 78 (9); 76 (7); 75 (5); 63 (7); 51 (9). HRMS [ESI(+),IT-TOF]: calculado $\left[\mathrm{C}_{16} \mathrm{H}_{15} \mathrm{~N}_{2} \mathrm{O}_{2}\right]^{+}$267,1128; encontrado 267,1318.

6-(piridin-2-il)-[1,3]dioxolano[4,5-g]quinolina 16: sólido marrom (0,63 mmol, 63\%), Tf = 158,3-158,5 ${ }^{\circ} \mathrm{C} . \mathrm{IV}\left(\mathrm{cm}^{-1}\right) \overline{\mathrm{v}}_{\max }: 3446,3067$, 2922, 1619, 1514, 1499, 1478, 1233, 1169, 1039, 851, 766, 740. RMN de ${ }^{1} \mathrm{H}\left(300 \mathrm{MHz}, \mathrm{CDCl}_{3}\right) \delta 6,09$ (s, 2H, H-2), 7,06 (dd, 1H, $J=0,42 \mathrm{~Hz}$ e $J=0,36 \mathrm{~Hz}, \mathrm{H}-4), 7,30$ (ddd, $1 \mathrm{H}, J=7,5 \mathrm{~Hz}, J=4,8$ Hz e $J=1,2 \mathrm{~Hz}, \mathrm{H}-5$ ') $, 7,44$ (dd, $1 \mathrm{H}, J=0,7 \mathrm{~Hz}$ e $J=0,42 \mathrm{~Hz}, \mathrm{H}-9$ ), 7,83 (ddd, $1 \mathrm{H}, J=8,0 \mathrm{~Hz}, J=7,5 \mathrm{~Hz}$ e $J=1,8 \mathrm{~Hz}, \mathrm{H}-4$ ') 8,06 (ddd, $1 \mathrm{H}, J=8,5 \mathrm{~Hz}, J=0,7 \mathrm{~Hz}$ e $J=0,36 \mathrm{~Hz}, \mathrm{H}-8), 8,37$ (d, $1 \mathrm{H}, J=8,5$ Hz, H-7), 8,55 (ddd, $1 \mathrm{H}, J=8,0 \mathrm{~Hz}, J=1,2 \mathrm{~Hz}$ e $J=0,9 \mathrm{~Hz}, \mathrm{H}_{-} 3^{\prime}$ ), 8,70 (ddd, $1 \mathrm{H}, J=4,8 \mathrm{~Hz}, J=1,8 \mathrm{~Hz}$ e $J=0,9 \mathrm{~Hz}, \mathrm{H}-6$ '). RMN de ${ }^{13} \mathrm{C}\left(75 \mathrm{MHz}, \mathrm{CDCl}_{3}\right) \delta 101,7$ (C-9), 102,7 (C-2), 106,1 (C-4), 117,3 (C-7), 121,4 (C-3'), 123,6 (C-5'), 125,3 (C-12), 135,5 (C-8), 136,8 (C-4'), 146,1 (C-13), 148,1 (C-6), 149,1 (C-6'), 150,8 (C-10), 154,1 (C-11), 156,5 (C-2'). EM, m/z (\%) $250\left(\mathrm{C}_{15} \mathrm{H}_{10} \mathrm{~N}_{2} \mathrm{O}_{2}\right)\left(\left[\mathrm{M}^{+}\right], 100\right)$, $251\left([\mathrm{M}+1]^{+}, 19\right), 249$ (41), 221 (7), 194 (5), 193 (9), 192 (13), 191 (8), 165 (5), 164 (9), 140 (6), 139 (5), 138 (6), 125 (5), 114 (10), 113 (5), 87 (7), 78 (11), 63 (9), 62 (9), 52 (11), 51 (25), 50 (14), 40 (5). HRMS [ESI(+),IT-TOF]: calculado $\left[\mathrm{C}_{15} \mathrm{H}_{11} \mathrm{~N}_{2} \mathrm{O}_{2}\right]^{+} 251,0815$; encontrado 251,0734 .

7-metoxi-2-(piridin-2-il)quinolina 17: sólido marrom (0,50 mmol, $50 \%), \mathrm{Tf}=105-106^{\circ} \mathrm{C} . \mathrm{IV}\left(\mathrm{cm}^{-1}\right) \overline{\mathrm{v}}_{\max }: 3063,2923,2852,1622,1509$, 1457, 1215, 1173, 1023, 845, 765, 671. RMN de ${ }^{1} \mathrm{H}(300 \mathrm{MHz}$, $\left.\mathrm{CDCl}_{3}\right) \delta 3,99\left(\mathrm{~s}, 3 \mathrm{H}, \mathrm{OCH}_{3}\right), 7,21$ (dd, $1 \mathrm{H}, J=8,9$ e $\left.J=2,5 \mathrm{~Hz}, \mathrm{H}-6\right)$, (ddd, $1 \mathrm{H}, J=7,5 \mathrm{~Hz}, J=4,8 \mathrm{~Hz}$ e $J=1,1 \mathrm{~Hz}, \mathrm{H}-5$ ') , 7,51 (d, 1H, $J$ $=2,5 \mathrm{~Hz}, \mathrm{H}-8), 7,74$ (d, 1H, $J=8,9 \mathrm{~Hz}, \mathrm{H}-5), 7,87$ (dt, $1 \mathrm{H}, J=7,7 \mathrm{e}$ $J=1,8 \mathrm{~Hz}, \mathrm{H}-4^{\prime}$ ), 8,21 (d, 1H, $\left.J=8,5 \mathrm{~Hz}, \mathrm{H}-4\right), 8,40$ (d, $1 \mathrm{H}, J=8,5$ Hz, H-3), 8,59 (ddd, $1 \mathrm{H}, J=7,7 \mathrm{~Hz}, J=1,1 \mathrm{~Hz}$ e $J=0,9$ Hz, H-3'), 8,74 (ddd, $1 \mathrm{H}, J=4,8 \mathrm{~Hz}, J=1,8 \mathrm{~Hz}$ e $J=0,9 \mathrm{~Hz}, \mathrm{H}-6$ '). RMN de ${ }^{13} \mathrm{C}\left(75 \mathrm{MHz}, \mathrm{CDCl}_{3}\right) \delta 55,5\left(\mathrm{OCH}_{3}\right), 107,6(\mathrm{C}-8), 116,9(\mathrm{C}-3), 120,0$ (C-6), 121,7 (C-3'), 123,5 (C-9), 123,9 (C-5'), 128,6 (C-5), 136,5 (C-4), 136,9 (C-4'), 149,2 (C-6'), 149,6 (C-10), 156,4 (C-2), 156,5 (C-2'), 160,8 (C-7). EM, m/z (\%) $236\left(\mathrm{C}_{15} \mathrm{H}_{12} \mathrm{~N}_{2} \mathrm{O}\right)\left(\left[\mathrm{M}^{+} \cdot\right], 100\right), 237$ $\left([\mathrm{M}+1]^{+}, 15\right), 221(16), 207$ (7), $206(5), 205$ (12), 193 (20), 192 (19), 118 (6), 78 (5), 51 (14), 50 (7). HRMS [ESI(+),IT-TOF]: calculado $\left[\mathrm{C}_{15} \mathrm{H}_{13} \mathrm{~N}_{2} \mathrm{O}\right]^{+}$237,1022; encontrado 237,0935.

2-(6-bromopiridin-2-il)-7-metoxiquinolina 18: sólido amarelo $(0,44 \mathrm{mmol}, 44 \%), \mathrm{Tf}=163-164^{\circ} \mathrm{C}$. IV $\left(\mathrm{cm}^{-1}\right) \overline{\mathrm{v}}_{\text {max }}: 3063,2934,2833$, $1625,1544,1463,1427,1219,1129,1035,850,778,712$. RMN de ${ }^{1} \mathrm{H}\left(300 \mathrm{MHz}, \mathrm{CDCl}_{3}\right) \delta 3,99\left(\mathrm{~s}, 3 \mathrm{H}, \mathrm{OCH}_{3}\right), 7,22$ (dd, $1 \mathrm{H}, J=8,8$, e $J=2,5 \mathrm{~Hz}, \mathrm{H}-6), 7,51$ (d, 1H, $J=2,5 \mathrm{~Hz}, \mathrm{H}-8), 7,53$ (dd, 1H, $J=$ 7,7 Hz e $J=0,6 \mathrm{~Hz}, \mathrm{H}-5$ '); 7,71 (t, 1H, $J=7,7 \mathrm{~Hz}, \mathrm{H}-4$ ') , 7,73 (d, $1 \mathrm{H}, J=8,8 \mathrm{~Hz}, \mathrm{H}-5), 8,20$ (d, $1 \mathrm{H}, J=8,5 \mathrm{~Hz}, \mathrm{H}-4), 8,41$ (d, $1 \mathrm{H}, J=$ $8,5 \mathrm{~Hz}, \mathrm{H}-3$ ), 8,63 (dd, $1 \mathrm{H}, J=7,7 \mathrm{~Hz}$ e $J=0,6 \mathrm{~Hz}, \mathrm{H}-3$ '). RMN de ${ }^{13} \mathrm{C}\left(75 \mathrm{MHz}, \mathrm{CDCl}_{3}\right) \delta 55,5$ (C-11), 107,5 (C-8), 116,9 (C-6) 120,3 (C-3), 120,4 (C-3'), 123,8 (C-9), 128,2 (C-5'), 128,6 (C-5), 136,6 (C-4'), 139,1 (C-4), 141,5 (C-10), 149,5 (C-6'), 154,7 (C-2), 157,6 (C-2'), 160,9 (C-7). EM, m/z (\%) $314\left(\mathrm{C}_{15} \mathrm{H}_{11} \mathrm{BrN}_{2} \mathrm{O}\right)\left(\left[\mathrm{M}^{+}\right], 49\right)$, $315\left([\mathrm{M}+1]^{+}, 9\right), 316\left([\mathrm{M}+2]^{+}, 43\right), 273(5), 236(17), 235$ (100), 207 (13), 205 (11), 193 (9), 192 (53), 191 (13), 165 (11), 164 (15), 158 (14), 140 (6), 138 (7), 128 (5), 118 (7), 117 (12), 115 (15), 114 (11), 89 (8), 88 (8), 76 (11), 75 (11), 63 (20), 62 (1), 52 (10), 51 (20), 50 (24). HRMS [ESI(+),IT-TOF]: calculado $\left[\mathrm{C}_{15} \mathrm{H}_{12} \mathrm{BrN}_{2} \mathrm{O}\right]^{+} 315,0128$; encontrado 314,9900. 
6-metoxi-2-(piridin-2-il)quinolina 19: sólido branco (0,61 mmol, $61 \%), \mathrm{Tf}=107-108^{\circ} \mathrm{C}$. IV $\left(\mathrm{cm}^{-1}\right) \overline{\mathrm{v}}_{\max }: 3065,2930,2834,1619,1496$, 1381, 1222, 1166, 1018, 866, 793, 629. RMN de ${ }^{1} \mathrm{H}(300 \mathrm{MHz}$, $\left.\mathrm{CDCl}_{3}\right) \delta 3,95\left(\mathrm{~s}, 3 \mathrm{H}, \mathrm{OCH}_{3}\right), 7,11(\mathrm{~d}, 1 \mathrm{H}, J=2,8 \mathrm{~Hz}, \mathrm{H}-5), 7,33$ (ddd, $1 \mathrm{H}, J=7,5 \mathrm{~Hz}, J=4,8 \mathrm{~Hz}$ e $J=1,2 \mathrm{~Hz}, \mathrm{H}-5$ '), 7,39 (dd, $1 \mathrm{H}$, $J=9,2$ e $J=2,8 \mathrm{~Hz}, \mathrm{H}-7$ ), 7,86 (dt, $1 \mathrm{H}, J=7,5$ e $J=1,8 \mathrm{~Hz}, \mathrm{H}-4$ '), $8,07$ (d, $1 \mathrm{H}, J=9,2 \mathrm{~Hz}, \mathrm{H}-8$ ), 8,17 (d, $1 \mathrm{H}, J=8,6 \mathrm{~Hz}, \mathrm{H}-4), 8,50$ (d, $1 \mathrm{H}, J=8,6 \mathrm{~Hz}, \mathrm{H}-3), 8,59$ (ddd, $1 \mathrm{H}, J=7,5 \mathrm{~Hz}, J=1,2 \mathrm{~Hz}$ e $J$ $=0,9 \mathrm{~Hz}, \mathrm{H}-3$ '), 8,71 (ddd, $1 \mathrm{H}, J=4,8 \mathrm{~Hz}, J=1,8 \mathrm{~Hz}$ e $J=0,9 \mathrm{~Hz}$, H-6'). RMN de ${ }^{13} \mathrm{C}\left(75 \mathrm{MHz}, \mathrm{CDCl}_{3}\right) \delta 55,6\left(\mathrm{OCH}_{3}\right), 105,1(\mathrm{C}-5)$, 119,3 (C-3), 121,6 (C-3'), 122,4 (C-7), 123,7 (C-5'), 129,3 (C-9), 131,1 (C-8), 135,6 (C-4), 137,0 (C-4'), 143,8 (C-10), 148,9 (C-6'), 153,7 (C-2), 156,3 (C-2'), 158,1 (C-6). EM m/z (\%) $236\left(\mathrm{C}_{15} \mathrm{H}_{12} \mathrm{~N}_{2} \mathrm{O}\right.$, $\left(\left[\mathrm{M}^{+}\right], 100\right),\left([\mathrm{M}+1]^{+}, 17\right), 221(22), 194(6), 193$ (44), 192 (30), 166 (7), 165 (5), 140 (6), 118 (6), 63 (9), 62 (6), $52(7), 51$ (16), 50 (8). HRMS [ESI(+),IT-TOF]: calculado $\left[\mathrm{C}_{15} \mathrm{H}_{13} \mathrm{~N}_{2} \mathrm{O}\right]^{+} 237,1022$; encontrado 237,0943 .

2-(piridin-2-il)quinolina 20: sólido branco (0,42 mmol, 42\%), Tf $=93-93,5^{\circ} \mathrm{C}$. IV $\left(\mathrm{cm}^{-1}\right) \overline{\mathrm{v}}_{\max }: 3060,2922,1594,1501,1122,845,776$, $740,620,481$. RMN de ${ }^{1} \mathrm{H}\left(300 \mathrm{MHz}, \mathrm{CDCl}_{3}\right) \delta 7,35$ (ddd, $1 \mathrm{H}, J=$ $7,5 \mathrm{~Hz}, J=4,8 \mathrm{~Hz}$ e $J=1,1 \mathrm{~Hz}, \mathrm{H}-5$ '); 7,55 (ddd, $1 \mathrm{H}, J=7,5 \mathrm{~Hz}, J$ $=6,9 \mathrm{~Hz}$ e $J=0,9 \mathrm{~Hz}, \mathrm{H}-6), 7,73$ (ddd, $1 \mathrm{H}, J=8,3 \mathrm{~Hz}, J=6,9 \mathrm{~Hz}$ e $J=1,3 \mathrm{~Hz}, \mathrm{H}-7$ ); 7,85 (dd, 1H, $J=7,5 \mathrm{~Hz}$ e $J=1,3 \mathrm{~Hz}, \mathrm{H}-5$ ); 7,88 (dt, $1 \mathrm{H}, J=7,4 \mathrm{~Hz}$ e $J=1,7 \mathrm{~Hz}, \mathrm{H}-4^{\prime}$ ); 8,19 (dd, $1 \mathrm{H}, J=8,3 \mathrm{~Hz}$ e $J$ $=0,9 \mathrm{~Hz}, \mathrm{H}-8) ; 8,28(\mathrm{~d}, 1 \mathrm{H}, J=8,6 \mathrm{~Hz}, \mathrm{H}-4) ; 8,56(\mathrm{~d}, 1 \mathrm{H}, J=8,6$ Hz, H-3); 8,66 (ddd, $1 \mathrm{H}, J=7,4 \mathrm{~Hz}, J=1,1 \mathrm{~Hz}$ e $J=0,6 \mathrm{~Hz}, \mathrm{H}-3^{\prime}$ ); 8,74 (ddd, $1 \mathrm{H}, J=4,8 \mathrm{~Hz}, J=1,7 \mathrm{~Hz}$ e $J=0,6 \mathrm{~Hz}, \mathrm{H}-6$ '). RMN de ${ }^{13} \mathrm{C}\left(75 \mathrm{MHz}, \mathrm{CDCl}_{3}\right) \delta 118,95$ (C-3), 121,83 (C-3'), 124,01 (C-5'), 126,74 (C-6), 127,60 (C-5), 128,23 (C-9), 129,54 (C-7), 129,70 (C-8), 136,80 (C-4), 136,94 (C-4'), 147,90 (C-10), 149,14 (C-6'), 156,14 (C-2), 156,31 (C-2'). EM m/z (\%) $206\left(\mathrm{C}_{14} \mathrm{H}_{10} \mathrm{~N}_{2},\left([\mathrm{M}]^{+}\right.\right.$, 100), ([M+1] $\left.]^{+}, 8\right) ; 205$ (11), 179 (15), 178 (29), 128 (21), 103 (18), 89 (14), 75 (16), 51 (24), 50 (15). HRMS [ESI(+),IT-TOF]: calculado $\left[\mathrm{C}_{14} \mathrm{H}_{11} \mathrm{~N}_{2}\right]^{+}$207,0917; encontrado 207,0594.

2-(6-bromopiridin-2-il)quinolina 21: sólido amarelo (0,40 mmol, $40 \%)(0,29 \mathrm{mmol}, 29 \%), \mathrm{Tf}=154-155^{\circ} \mathrm{C} . \mathrm{IV}\left(\mathrm{cm}^{-1}\right) \overline{\mathrm{v}}_{\text {max }}: 2922,2852$, $1715,1595,1544,1444,1117,986,783,730$. RMN de ${ }^{1} \mathrm{H}(300 \mathrm{MHz}$, $\left.\mathrm{CDCl}_{3}\right) \delta 7,52(\mathrm{~d}, J=7,8 \mathrm{~Hz}, 1 \mathrm{H}, \mathrm{H}-5$ '); 7,55 (t, $1 \mathrm{H}, J=8,1 \mathrm{~Hz}, \mathrm{H}-6)$; 7,70 (t, $1 \mathrm{H}, J=7,8 \mathrm{~Hz}, \mathrm{H}-4$ ') ; 7,73 (dt, $1 \mathrm{H}, J=8,1 \mathrm{~Hz}$ e $J=0,7 \mathrm{~Hz}$ H-7); 7,84 (dd, $1 \mathrm{H}, J=8,1 \mathrm{~Hz}$ e $J=0,7 \mathrm{~Hz}, \mathrm{H}-5) ; 8,15$ (d, 1H, $J=$ $8,5 \mathrm{~Hz}, \mathrm{H}-8) ; 8,26$ (d, $1 \mathrm{H}, J=8,6 \mathrm{~Hz}, \mathrm{H}-4) ; 8,55$ (d, $1 \mathrm{H}, J=8,6 \mathrm{~Hz}$, $\mathrm{H}-3)$; 8,63 (d, $1 \mathrm{H}, J=7,8 \mathrm{~Hz}, \mathrm{H}-3$ '). RMN de ${ }^{13} \mathrm{C}\left(75 \mathrm{MHz}, \mathrm{CDCl}_{3}\right)$ $\delta$ 119,00 (C-3), 120,42 (C-3'), 127,04 (C-5'), 127,63 (C-7), 128,28 (C-8), 128,42 (C-9), 129,67 (C-6), 129,80 (C-5), 136,87 (C-4), 139, 16 (C-4'), 141,50 (C-10), 147,81 (C-6'), 154,44 (C-2), 157,46 (C-2'). $\mathrm{EM} m / z(\%) 285\left(\mathrm{C}_{14} \mathrm{H}_{9} \mathrm{BrN}_{2},\left([\mathrm{M}]^{+}, 12\right),\left([\mathrm{M}+1]^{+}, 55\right) ; 284\right.$ (55), 206 (16), 205 (100), 128 (36), 102 (16), 89 (14). HRMS [ESI(+),IT-TOF]: calculado $\left[\mathrm{C}_{15} \mathrm{H}_{10} \mathrm{BrN}_{2}\right]^{+}$285,0022; encontrado 285,0276.

6,8-dimetoxi-2-(piridin-2-il)quinolina 22: sólido amarelo $(0,50$ mmol, 50\%), Tf $=82-83^{\circ} \mathrm{C}$. IV $\left(\mathrm{cm}^{-1}\right) \overline{\mathrm{v}}_{\max }: 2933,1617,1588,1451$, 1214, 1158, 1044, 779, 486. RMN de ${ }^{1} \mathrm{H}\left(300 \mathrm{MHz}, \mathrm{CDCl}_{3}\right) \delta 3,93$ (s, 3H, $\left.\mathrm{OCH}_{3}\right) ; 4,08$ (s, 3H, $\left.\mathrm{OCH}_{3}\right) ; 6,70(\mathrm{~d}, 1 \mathrm{H}, J=2,5 \mathrm{~Hz}, \mathrm{H}-5)$; $6,73(\mathrm{~d}, 1 \mathrm{H}, J=2,5 \mathrm{~Hz}, \mathrm{H}-7) ; 7,30$ (ddd, $1 \mathrm{H}, J=7,4 \mathrm{~Hz}, J=4,8 \mathrm{~Hz}$ e $J=1,1 \mathrm{~Hz}, \mathrm{H}-5^{\prime}$ ); 7,83 (dt, 1H, $J=7,4 \mathrm{~Hz}$ e $J=1,8 \mathrm{~Hz}, \mathrm{H}-4$ '); 8,13 (d, $1 \mathrm{H}, J=8,6 \mathrm{~Hz}, \mathrm{H}-4) ; 8,53$ (d, $1 \mathrm{H}, J=8,6 \mathrm{~Hz}, \mathrm{H}-3$ ); 8,64 (ddd, $1 \mathrm{H}, J=7,4 \mathrm{~Hz}, J=1,1 \mathrm{~Hz}$ e $J=0,8 \mathrm{~Hz}, \mathrm{H}-3$ '); 8,68 (ddd, $1 \mathrm{H}, J=4,8$ $\mathrm{Hz}, J=1,8 \mathrm{~Hz}$ e $J=0,8 \mathrm{~Hz}, \mathrm{H}-6$ '). RMN de ${ }^{13} \mathrm{C}\left(75 \mathrm{MHz}, \mathrm{CDCl}_{3}\right.$ ) $\delta$ 55,52 (OMe), 56,19 (OMe), 97,01 (C-7), 101,49 (C-5), 119,90 (C-3), 121,72 (C-5'), 123,58 (C-3'), 130,11 (C-9), 135,61 (C-4'), 136,40 (C-6), 136,85 (C-4), 148,94 (C-3'), 152,81 (C-8), 156,44
(C-10), 156,51 (C-2), 158,62 (C-2'). EM m/z (\%) $266\left(\mathrm{C}_{16} \mathrm{H}_{14} \mathrm{~N}_{2} \mathrm{O}_{2}\right.$, $\left([\mathrm{M}]^{+}, 79\right),\left([\mathrm{M}+1]^{+}, 15\right) ; 265(100), 237(40), 236(25), 193(21), 179$ (14), 104 (14), 97 (17), 78 (16), 51 (18). HRMS [ESI(+),IT-TOF]: calculado $\left[\mathrm{C}_{16} \mathrm{H}_{15} \mathrm{~N}_{2} \mathrm{O}_{2}\right]^{+}$267,1128; encontrado 267,0615.

6-(6-bromopiridin-2-il)-[1,3]dioxolano[4,5-g]quinolina 23: sólido amarelo $(0,49 \mathrm{mmol}, 49 \%), \mathrm{Tf}=128-129^{\circ} \mathrm{C}$. IV $\left(\mathrm{cm}^{-1}\right) \overline{\mathrm{v}}_{\max }$ : 2919, 2850, 1717, 1522, 1462, 1259, 1123, 1037, 794. RMN de ${ }^{1} \mathrm{H}\left(300 \mathrm{MHz}, \mathrm{CDCl}_{3}\right) \delta 6,13\left(\mathrm{~s}, 2 \mathrm{H}, \mathrm{CH}_{2}\right) ; 7,09$ (s, 1H, H-5); 7,42 (s, 1H, H-8); 7,50 (d, $J=7,7 \mathrm{~Hz}, 1 \mathrm{H}, \mathrm{H}-5$ '); 7,69 (t, $J=7,7 \mathrm{~Hz}$, 1H, H-4'); 8,07 (d, $1 \mathrm{H}, J=8,5 \mathrm{~Hz}, \mathrm{H}-4) ; 8,38$ (d, $1 \mathrm{H}, J=8,5 \mathrm{~Hz}$, $\mathrm{H}-3)$; 8,55 (d, $1 \mathrm{H}, J=7,7 \mathrm{~Hz}, \mathrm{H}-3$ '). RMN de ${ }^{13} \mathrm{C}\left(75 \mathrm{MHz}, \mathrm{CDCl}_{3}\right)$ $\delta$ 101,77 (C-11), 102,67 (C-5), 106,05 (C-8), 112,79 (C-3), 117,46 (C-9), 119,99 (C-3'), 125,07 (C-10), 127,82 (C-5'), 135,59 (C-4), 139,10 (C-4'), 140,70 (C-6'), 145,34 (C-2), 148,36 (C-6), 151,34 (C-7), 156,66 (C-2'). EM m/z (\%) $329\left(\mathrm{C}_{15} \mathrm{H}_{9} \mathrm{BrN}_{2} \mathrm{O}_{2}\right.$, ([M] $\left.{ }^{+}, 27\right)$, ([M+1] $\left.]^{+}, 99\right) ; 328$ (98), 250 (18), 249 (100), 191 (21), 164 (16), 124 (31). HRMS [ESI(+),IT-TOF]: calculado $\left[\mathrm{C}_{15} \mathrm{H}_{10} \mathrm{BrN}_{2}\right]^{+} 328,992$; encontrado 329,0203 .

8-metil-2-(piridin-2-il)quinolina 24: sólido branco $(0,30 \mathrm{mmol}$, $30 \%), \mathrm{Tf}=80,5-81^{\circ} \mathrm{C} . \mathrm{IV}\left(\mathrm{cm}^{-1}\right) \overline{\mathrm{v}}_{\text {max }}: 2917,1590,1476,1040,847$, 776, 592, 404. RMN de ${ }^{1} \mathrm{H}\left(300 \mathrm{MHz}, \mathrm{CDCl}_{3}\right) \delta 2,92$ (s, 3H, $\left.\mathrm{CH}_{3}\right)$; 7,35 (ddd, $1 \mathrm{H}, J=7,8 \mathrm{~Hz}, J=4,8 \mathrm{~Hz}$ e $J=1,2, \mathrm{H}-5$ '); 7,43 (t, 1H, $J=7,3 \mathrm{~Hz}, \mathrm{H}-6) ; 7,58$ (d, $J=7,3 \mathrm{~Hz}, 1 \mathrm{H}, \mathrm{H}-7) ; 7,69$ (d, $J=7,3 \mathrm{~Hz}$, $1 \mathrm{H}, \mathrm{H}-5) ; 7,87$ (dt, $J=7,8 \mathrm{~Hz}$ e $\left.J=1,8 \mathrm{~Hz}, 1 \mathrm{H}, \mathrm{H}-4^{\prime}\right) ; 8,25$ (d, $J=$ $8,6 \mathrm{~Hz}, 1 \mathrm{H}, \mathrm{H}-4) ; 8,59$ (d, $J=8,6 \mathrm{~Hz}, 1 \mathrm{H}, \mathrm{H}-3) ; 8,71-8,78$ (m, 2H, H-6' e H-3'). RMN de ${ }^{13} \mathrm{C}\left(75 \mathrm{MHz}, \mathrm{CDCl}_{3}\right) \delta 17,85\left(\mathrm{CH}_{3}\right), 118,36$ (C-3), 121,80 (C-3'), 123,87 (C-5'), 125,54 (C-5), 126,52 (C-6), 128,19 (C-8), 129,57 (C-7), 136,87 (C-4'), 136,98 (C-4), 137,65 (C-9), 146,79 (C-10), 148,95 (C-6'), 154,58 (C-2)*, 156,68 (C-2'). $\mathrm{EM} m / z(\%) 220\left(\mathrm{C}_{15} \mathrm{H}_{12} \mathrm{~N}_{2},\left([\mathrm{M}]^{+}, 100\right),\left([\mathrm{M}+1]^{+}, 27\right) ; 219(40) ; 192\right.$ (11);115 (12); 110 (15); 51 (15). HRMS [ESI(+),IT-TOF]: calculado $\left[\mathrm{C}_{15} \mathrm{H}_{13} \mathrm{~N}_{2}\right]^{+} 221,1073$; encontrado 221,1016.

2-(6-bromopiridin-2-il)-8-metilquinolina 25: sólido amarelo (0,29 mmol, 29\%), Tf $=108-109^{\circ} \mathrm{C} . \mathrm{IV}\left(\mathrm{cm}^{-1}\right) \overline{\mathrm{v}}_{\text {max }}: 3046,2920,1549,1418$, $1128,1073,846,788,749$. RMN de ${ }^{1} \mathrm{H}\left(300 \mathrm{MHz} \mathrm{CDCl}_{3}\right) \delta 2,89$ (s, 3H, $\left.\mathrm{CH}_{3}\right) ; 7,44$ (t, $\left.1 \mathrm{H}, J=7,6 \mathrm{~Hz}, \mathrm{H}-6\right) ; 7,52(\mathrm{~d}, J=7,7 \mathrm{~Hz}, 1 \mathrm{H}$, H-5'); 7,58 (dd, $1 \mathrm{H}, J=7,6 \mathrm{~Hz}$ e $J=1,8 \mathrm{~Hz}, \mathrm{H}-7$ ); 7,67 (dd, $1 \mathrm{H}, J=$ $7,6 \mathrm{~Hz}$ e $J=1,8 \mathrm{~Hz}, \mathrm{H}-5$ ); 7,70 (t, 1H, $J=7,7 \mathrm{~Hz}, \mathrm{H}-4$ '); 8,23 (d, $J$ $=8,60 \mathrm{~Hz}, \mathrm{H}-4) ; 8,55(\mathrm{~d}, 1 \mathrm{H}, J=8,60 \mathrm{~Hz}, \mathrm{H}-3) ; 8,70(\mathrm{~d}, 1 \mathrm{H}, J=7,7$ $\mathrm{Hz}, \mathrm{H}-3^{\prime}$ ). RMN de ${ }^{13} \mathrm{C}\left(75 \mathrm{MHz}, \mathrm{CDCl}_{3}\right) \delta 17,81$ (C-11), 118,48 (C-3), 120,36 (C-3'), 125,56 (C-5'), 126,84 (C-7), 128,07 (C-6), 128,39 (C-9), 129,69 (C-5), 137,06 (C-4), 137,69 (C-8), 139,07 (C4'), 141,41 (C-10), 146,72 (C-6'), 152,98 (C-2), 157,88 (C-2'). EM $\mathrm{m} / \mathrm{z}(\%) 299\left(\mathrm{C}_{15} \mathrm{H}_{11} \mathrm{BrN}_{2},\left([\mathrm{M}]^{+}, 21\right),\left([\mathrm{M}+2]^{+}, 17\right) ; 219(53), 218\right.$ (11), 217 (13), 115 (14), 109 (17), 96 (23). HRMS [ESI(+),IT-TOF]: calculado $\left[\mathrm{C}_{15} \mathrm{H}_{12} \mathrm{BrN}_{2}\right]^{+}$299,0178; encontrado 299,0057.

2-(3-bromo-4-metoxifenil)-6,8-dimetoxiquinolina 26: sólido branco $(0,83 \mathrm{mmol}, 83 \%), \mathrm{Tf}=97-98^{\circ} \mathrm{C}$. IV $\left(\mathrm{cm}^{-1}\right) \overline{\mathrm{V}}_{\max }: 2934$, 2834, 2029, 1696, 1615, 1454, 1260, 1159, 1041, 842, 791, 670, 661. RMN de ${ }^{1} \mathrm{H}\left(300 \mathrm{MHz}, \mathrm{CDCl}_{3}\right) \delta 3,92$ (s, 3H, H-13); 3,95 (s, $3 \mathrm{H}, \mathrm{H}-11$ ); 4,06 (s, 3H, H-12); 6,66 (d, 1H, J = 2,5 Hz, H-5); 6,72 (d, $1 \mathrm{H}, J=2,5 \mathrm{~Hz}, \mathrm{H}-7) ; 6,99$ (d, 1H, $J=8,6 \mathrm{~Hz}, \mathrm{H}-5$ '); 7,78 (d, 1H, $J=8,6 \mathrm{~Hz}, \mathrm{H}-3$ ); 8,04 (d, 1H, $J=8,6 \mathrm{~Hz}, \mathrm{H}-4$ ); 8,07 (dd, $1 \mathrm{H}, J=8,6$ Hz e $J=2,7$ Hz, H-6'); 8,38 (d, 1H, $J=2,7 \mathrm{~Hz}, \mathrm{H}-2^{\prime}$ ). RMN de ${ }^{13} \mathrm{C}$ $\left(75 \mathrm{MHz}, \mathrm{CDCl}_{3}\right) \delta$ 55,76 (C-11); 56,40 (C-13); 56,57 (C-12); 97,03 (C-5); 101,78 (C-7); 111,97 (C-5'); 112,35 (C-3'); 119,43 (C-3); 127,65 (C-6'); 128,98 (C-9); 132,36 (C-2'); 133,95 (C-1'); 135,93 (C-4); 136,89 (C-10); 152,29 (C-2); 156,57 (C-6); 156,67 (C-8); 158,38 (C-4'). EM m/z (\%) $373\left(\mathrm{C}_{18} \mathrm{H}_{16} \mathrm{BrNO}_{3},\left([\mathrm{M}]^{+}, 90\right),\left([\mathrm{M}+2]^{+}\right.\right.$, 
100); 346 (40); 345 (21); 344 (49); 178 (16); 165 (27); 103 (10); 96 (11); 89 (16); 82 (11); 75 (17). HRMS [ESI(+),IT-TOF]: calculado $\left[\mathrm{C}_{18} \mathrm{H}_{17} \mathrm{BrNO}_{3}\right]^{+}$374,0386; encontrado 374,0290.

\section{Ensaios biológicos}

\section{Avaliação da atividade antifúngica}

Para a avaliação da atividade antifúngica dos compostos 5-8 (Figura 1), disponíveis no laboratório, e os recém preparados 12-26 (Esquema 1), utilizou-se o método da microdiluição em placa de poliestireno de 96 poços, conforme proposto pelo documento M27-A2 do Clinical and Laboratory Standards Institute. ${ }^{36}$ Os bioensaios foram conduzidos com os fungos patogênicos oportunistas Candida albicans (ATCC 22019), Candida glabrata (ATCC 90030), Candida tropicalis (ATCC 750), Candida parapsilosis (ATCC 22019), Candida krusei (ATCC 20029) e com a levedura Cryptococcus neoformans (ATCC 24067) pertencentes à coleção de cultura de micro-organismos do Laboratório de Biotecnologia e Bioensaios do Instituto de Ciências Exatas da Universidade Federal de Minas Gerais (ICEx-UFMG).

Os micro-organismos foram incubados em estufa a $37^{\circ} \mathrm{C}$ por 36 h. As suspensões contendo os micro-organismos foram transferidas para tubos contendo água destilada estéril. Os tubos foram homogeneizados e a concentração ajustada para atingir uma suspensão (inóculo) compatível com o padrão 0,5 da escala de MacFarland $\left(10^{6}\right.$ células $\left.\mathrm{mL}^{-1}\right)$.

Uma solução inicial de cada composto (5-8 e 12-26) foi preparada utilizando-se como solvente o dimetilsulfóxido (DMSO) e o meio de cultura Sabouraud como diluente. Foram feitas diluições seriadas de modo que as concentrações testadas dos compostos foram 250,00, $125,00,62,50,31,25,15,63,7,81,3,90$ e $1,95 \mu \mathrm{g} \mathrm{mL}^{-1}$ em cada série da microplaca.

Quatro experimentos-controle foram feitos: (i) controle de crescimento do micro-organismo, para verificar a viabilidade celular; (ii) o branco, em que não se adicionou o inóculo, para eliminar o efeito da coloração da solução da substância teste; (iii) controle positivo, onde a solução-trabalho foi substituída pelos antifúngicos comerciais (miconazol ou nistatina); (iv) controle de esterilidade do meio de cultura, contendo $100 \mu \mathrm{L}$ de meio de cultura e $100 \mu \mathrm{L}$ de água destilada estéril.
As microplacas foram incubadas por 24 horas a $37{ }^{\circ} \mathrm{C}$. Ao final da incubação, o crescimento dos micro-organismos foi quantificado por densidade óptica através da absorbância a $530 \mathrm{~nm}$ usando um leitor de microplacas para avaliar os efeitos dos compostos testados sobre o crescimento fúngico. Avaliou-se a inibição de crescimento para todos os compostos na concentração de $250 \mu \mathrm{g} \mathrm{mL}^{-1}$. Os valores de $\mathrm{IC}_{50}$ foram calculados para as amostras que apresentaram inibição superior a $50 \%$ na concentração mais elevada. $\mathrm{O}$ experimento foi realizado em duplicata.

\section{RESULTADOS E DISCUSSÃO}

\section{Síntese de 2-(2-piridil)quinolinas}

Com vistas a ampliar a aplicabilidade da metodologia para a síntese de 2-(2-piridil)quinolinas relatada anteriormente, ${ }^{31} \mathrm{o}$ efeito do uso de radiação de micro-ondas na promoção da reação de Povarov entre anilina, 2-piridinocarbaldeido e etil vinil éter foi investigado. Para otimizar as condições reacionais foram empregados os reagentes e solventes apresentados na Tabela 1 .

Como catalisador foi utilizado $30 \mathrm{~mol} \%$ de trifluoreto de boro $\left(\mathrm{BF}_{3} . \mathrm{O}\left(\mathrm{CH}_{3}\right)_{2}\right)$, conforme estudo anterior. ${ }^{31} \mathrm{~A}$ reação foi realizada com nove solventes de polaridades diversas, sendo que os correspondentes aos experimentos 2, 4-9 (cf. Tabela 1) não foram previamente investigados. ${ }^{31}$

Como observado na Tabela 1, os melhores rendimentos (48-51\%) foram alcançados com etanol, acetonitrila e tetraidrofurano (Tabela 1, experimentos, 1, 3 e 9, respectivamente). Os rendimentos menores foram observados com o uso de solventes menos polares como diclorometano (Tabela 1, experimento 4) e éter dietílico (Tabela 1, experimento 7). Foi também realizada reação na ausência de solvente, todavia nesse caso o rendimento obtido foi muito baixo (Tabela 1, experimento 10). Em função desses resultados, acetonitrila foi selecionada para fins de otimização de outras condições reacionais. Deve-se notar que embora no presente caso o melhor rendimento tenha sido obtido com acetonitrila (51\%), bem inferior ao relatado com o uso de aquecimento convencional (81\%), o tempo de reação estabelecido nesse trabalho foi de apenas 20 minutos, em contraste com 24 horas no caso do estudo anterior. ${ }^{31}$

Na sequência, foi avaliado conjuntamente o efeito da concentração

Tabela 1. Efeito do solvente na síntese da quinolina $\mathbf{1 2}$ empregando a reação de Povarov

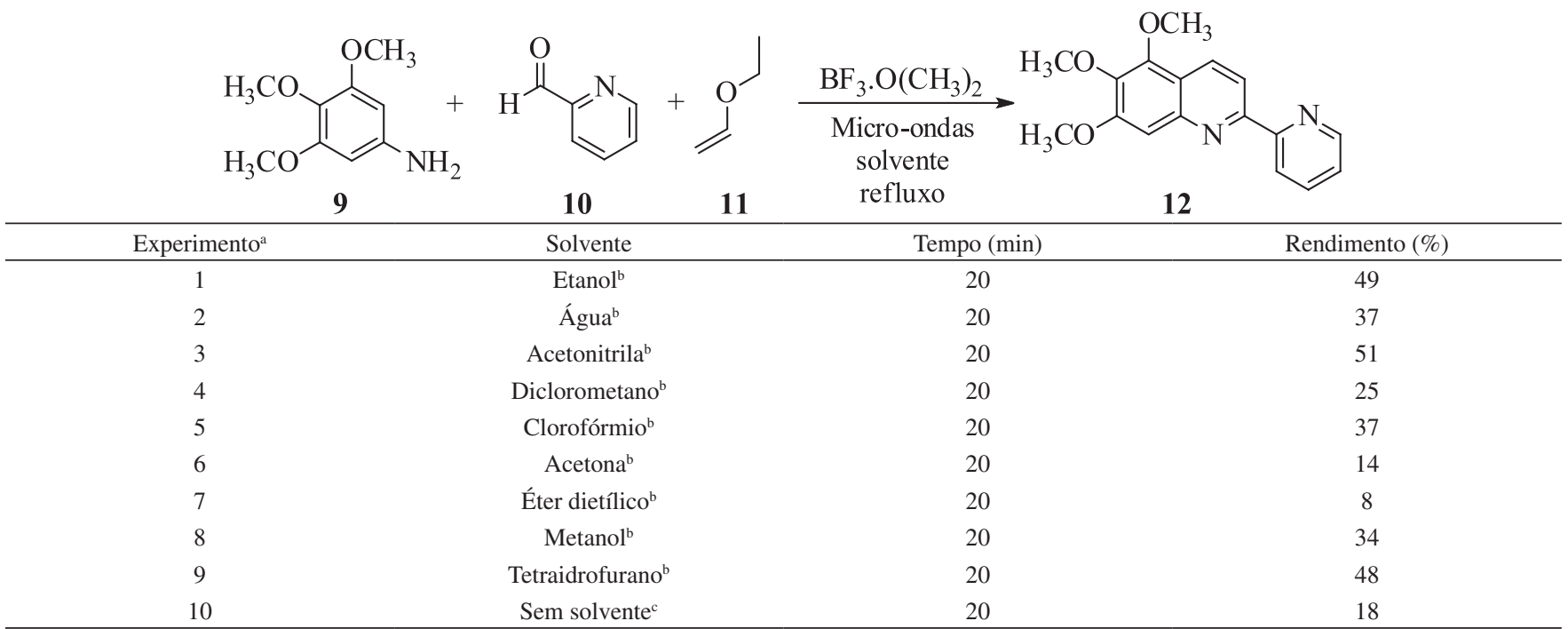

${ }^{a}$ Condições: 3,4,5-trimetoxianilina 9 (1,0 mmol), 2-piridinocarbaldeído $\mathbf{1 0}(1,0 \mathrm{mmol})$, etil vinil éter $\mathbf{1 1}(1,5 \mathrm{mmol}), \mathrm{BF} \cdot \mathrm{O}\left(\mathrm{CH}_{3}\right)_{2}(30 \mathrm{~mol} \%) ; 50 \mathrm{~W}$; ${ }^{\mathrm{b}} \mathrm{Refluxo}$.

${ }^{\mathrm{C}} 95{ }^{\circ} \mathrm{C}$. O progresso da reação foi monitorizado por cromatografia em camada delgada. 
do catalisador, do tempo de reação e da potência do micro-ondas sobre o rendimento do produto (Tabela 2).

Tabela 2. Efeito da quantidade do catalisador, tempo de reação e potência do micro-ondas no rendimento da quinolina $\mathbf{1}$, conforme esquema apresentado na Tabela 1

\begin{tabular}{ccccc}
\hline Experimento $^{\mathrm{a}}$ & $\begin{array}{c}\mathrm{BF}_{3} \cdot \mathrm{O}\left(\mathrm{CH}_{3}\right)_{2} \\
(\mathrm{~mol} \%)\end{array}$ & $\begin{array}{c}\text { Potência } \\
(\mathrm{W})\end{array}$ & $\begin{array}{c}\text { Tempo } \\
(\mathrm{min} .)\end{array}$ & $\begin{array}{c}\text { Rendimento } \\
(\%)\end{array}$ \\
\hline 1 & 10 & 50 & 20 & 32 \\
2 & 20 & 50 & 20 & 72 \\
3 & 30 & 50 & 20 & 52 \\
4 & 50 & 50 & 20 & 59 \\
5 & 100 & 50 & 20 & 44 \\
6 & 20 & 50 & 10 & 53 \\
7 & 20 & 50 & 15 & 66 \\
8 & 20 & 50 & 30 & 53 \\
9 & 20 & 50 & 60 & 71 \\
10 & 20 & 50 & 90 & 82 \\
11 & 20 & 50 & 120 & 88 \\
12 & 20 & 100 & 120 & 79 \\
13 & 20 & 150 & 120 & 0 \\
\hline
\end{tabular}

aCondições: 3,4,5-trimetoxianilina 9 (1,0 mmol), 2-piridinocarbaldeído $\mathbf{1 0}$ $(2,0 \mathrm{mmol})$, etil vinil éter $11(3,0 \mathrm{mmol})$, acetonitrila; $\mathrm{BF}_{3} \cdot \mathrm{O}\left(\mathrm{CH}_{3}\right)_{2}(20 \mathrm{~mol} \%)$; $50 \mathrm{~W} ; 95^{\circ} \mathrm{C}$.

Mantendo-se o tempo de reação constante (Tabela 2, experimentos 1-5), observou-se maior rendimento com o emprego de $20 \mathrm{~mol} \%$ do catalisador (Tabela 2, experimento 2). Tendo sido essa a condição de maior rendimento, foram feitas reações em concentração constante do catalisador e com variação de tempo (Tabela 2, experimento 6-11). O melhor rendimento (88\%), nessas condições, foi alcançado após 2 horas de reação (Tabela 2, experimento 11).

Finalmente foi avaliado o efeito da potência da radiação sobre o rendimento do produto desejado (Tabela. 2, experimentos 12 e 13). Para isso, foram empregadas as condições reacionais do experimento 11 (Tabela 2), utilizando-se potência de $100 \mathrm{~W}$ e $150 \mathrm{~W}$. Verifica-se pela Tabela 2 (experimentos 12 e 13) que o aumento na potência tem efeito restritivo sobre o rendimento da reação. Isso provavelmente se deve à decomposição dos reagentes, conforme observado pela análise da mistura reacional por cromatografia em camada delgada, que revelou a formação de uma mistura complexa marrom escura. Pelos resultados das Tabelas 1 e 2, ficou estabelecido que as melhores condições reacionais foram: uso de $20 \mathrm{~mol} \%$ de $\mathrm{BF}_{3} . \mathrm{O}\left(\mathrm{CH}_{3}\right)_{2}$ como catalisador; acetonitrila como solvente; 2 horas de reação a $95{ }^{\circ} \mathrm{C}$; irradiação sob a potência de $50 \mathrm{~W}$.

Uma vez estabelecidas as condições otimizadas para a reação sob micro-ondas (Tabela 2, experimento 11), e visando aumentar o escopo desse procedimento, as quinolinas 12-26 foram preparadas de acordo com metodologia apresentada no Esquema 1. Nesse esquema são também apresentados, para fins de comparação, os rendimentos dos produtos obtidos empregando-se as condições reacionais com aquecimento convencional. ${ }^{31}$

Pelos resultados (Esquema 1), verifica-se que no caso dos compostos $\mathbf{1 3}, \mathbf{1 4}, 15$ e 26, os rendimentos obtidos por aquecimento convencional e por micro-ondas foram praticamente iguais. Deve-se observar que o composto $\mathbf{2 6}$ foi obtido a partir de um benzaldeído substituído e não de uma piridina, indicando assim que a reação tem um escopo mais amplo. Apesar dos resultados praticamente iguais em termos de rendimento, o uso de micro-ondas como fonte de aquecimento tem a vantagem de reduzir o tempo reacional de 24 horas para apenas 2 horas.

Para os compostos 16, 17, 18 e 19, foi observado aumento nos rendimentos com o uso de micro-ondas. Adicionalmente, os compostos 20-25, que não haviam sido obtidos por meio de aquecimento convencional, foram preparados com sucesso com o uso de micro-ondas, embora com rendimentos moderados.

Todos os compostos tiveram suas estruturas elucidadas pelas análises dos espectros no infravermelho, $\mathrm{RMN}$ de ${ }^{1} \mathrm{H}$ e de ${ }^{13} \mathrm{C}$, e de massas. Experimentos bidimensionais de RMN (COSY, HSQC e $H M B C$ ) também foram realizados em alguns casos para fins de atribuição dos sinais de ${ }^{13} \mathrm{C}$ e de ${ }^{1} \mathrm{H}$ (ver Material Suplementar para todos os espectros). Os espectros no infravermelho de todos os compostos apresentaram bandas típicas de estiramento $\mathrm{C}=\mathrm{C}$ de anel aromático em torno de $1595-1615 \mathrm{~cm}^{-137}$ De um modo geral, para todos os compostos, os sinais dos hidrogênios H-3 e H-4 aparecem como dois dupletos ( $J$ $\simeq 8,6 \mathrm{~Hz}$ ) em aproximadamente $\delta=8,26$ e 8,54 , respectivamente. Os sinais das metoxilas e das metilas aparecem em torno de $\delta=3,95$ e 2,90 , respectivamente. As posições e multiplicidades dos sinais dos demais hidrogênios variaram em função do padrão de substituição e as atribuições foram confirmadas com bases das correlações do mapa de contorno COSY (ver figuras Material Suplementar). Para as atribuições dos sinais dos espectros de $\mathrm{RMN}$ de ${ }^{13} \mathrm{C}$ foi fundamental a análise dos mapas de contorno HSQC e HMBC, uma vez que diversos sinais apareceram com valores de deslocamentos químicos muito próximos. Por exemplo, no caso do composto 17, os sinais de C-4 e C-4' foram observados em $\delta=136,5$ e 136,9, respectivamente. As correlações com os sinais em $\delta=8,21(\mathrm{H}-4)$ e $\delta=7,87$ (H-4') permitiram a atribuições inequívoca dos sinais (Figura S15, Material Suplementar).

\section{Avaliação da atividade antifúngica}

Os compostos 5-8 e 12-26 foram submetidos a ensaios in vitro para avaliação da atividade contra C. albicans, C. glabrata, C. tropicalis, $C$. parapsilosis, $C$. krusei, fungos patogênicos frequentemente associados às infecções relatadas em unidades hematológicas e de tratamento intensivo. Também foi avaliada a atividade contra C. neoformans, levedura oportunista de relevância clínica por acometer pacientes pós-cirúrgicos e imunocomprometidos. Inicialmente foi feita uma avaliação das atividades dos compostos na dose de $250 \mu \mathrm{g} \mathrm{mL}^{-1}$, conforme resultados apresentados na Tabela 3.

Os produtos comerciais miconazol e nistatina foram utilizados como controle positivos, na mesma concentração das quinolinas avaliadas. Pelos dados obtidos (Tabela 3), verifica-se que miconazol e nistatina inibem completamente o crescimento das espécies de Candida spp., enquanto causam 71,9\% e 83,3\% de inibição no desenvolvimento de $C$. neoformans, respectivamente. Das quinolinas avaliadas, a maioria causou mais de $50 \%$ de inibição em pelo menos uma espécie de micro-organismo. As exceções foram os compostos $5,7,13,14,18$ e 26, sendo este último o menos ativo dentre todos. Embora esses resultados indiquem que o padrão e a natureza dos grupos substituintes nos compostos sejam importantes para as atividades, a total inatividade de $\mathbf{2 6}$ sugere que a presença do anel piridínico também seja necessária para a atividade biocida. Entretanto, para se comprovar essa hipótese seria necessário preparar e avaliar o composto análogo ao $\mathbf{2 6}$, contendo o anel piridínico.

O efeito de substituintes no anel piridina foi avaliado comparando as atividades dos pares de compostos halogenados e não halogenados no anel piridina $(5 / 12 ; 6 / 15 ; 8 / 19 ; 14 / 13 ; 18 / 17 ; 21 / 20 ; 23 / 16 ; 25 / 24)$ (Tabela 3). Não foi possível estabelecer correlação direta de efeito do halogênio sobre a atividade, uma vez que ocorreram aumento e diminuição da atividade quando se considera uma mesma cepa. Por outro lado, a análise em conjunto de atividade contra Candida spp. e $C$. neoformans mostrou que os compostos 12, 20 e $\mathbf{2 4}$ são mais ativos em relação aos seus análogos halogenados 5, 21 e 25, em todos os casos.

Contrariamente ao observado sobre o efeito dos substituintes 
<smiles>COc1cc2nc(-c3ccccn3)ccc2c(OC)c1OC</smiles><smiles>COc1ccc(OC)c2nc(-c3ccccn3)ccc12</smiles>

$13(76 \%, 79 \%)$<smiles>COc1ccc2ccc(-c3ccccn3)nc2c1</smiles>

$17(31 \%, 50 \%)$<smiles>Brc1cccc(-c2ccc3ccccc3n2)n1</smiles>

$21(0,40 \%)$<smiles>Cc1cccc2ccc(-c3cccc(Br)n3)nc12</smiles>

$25(0,29 \%)$<smiles>COc1ccc(OC)c2nc(-c3cccc(Br)n3)ccc12</smiles>

$14(61 \%, 61 \%)$<smiles>COc1ccc2ccc(-c3cccc(Br)n3)nc2c1</smiles>

$18(37 \%, 44 \%)$<smiles>COc1cc(OC)c2nc(-c3ccccn3)ccc2c1</smiles>

$22(0,50 \%)$<smiles>COc1cc2ccc(-c3ccccn3)nc2cc1OC</smiles>

$15(73 \%, 73 \%)$<smiles>COc1ccc2nc(-c3ccccn3)ccc2c1</smiles>

$19(32 \%, 61 \%)$<smiles>Brc1cccc(-c2ccc3cc4c(cc3n2)OCO4)n1</smiles>

$23(0,49 \%)$<smiles>c1ccc(-c2ccc3cc4c(cc3n2)OCO4)nc1</smiles>

$16(48 \%, 63 \%)$
$20(0,42 \%)$<smiles>Cc1cccc2ccc(-c3ccccn3)nc12</smiles>

$24(0,30 \%)$

${ }^{a}$ Rendimento de reação sob aquecimento convencional (Borel et al., 2015)

${ }^{b}$ Rendimento de reação sob aquecimento micro-ondas.

Esquema 1. Síntese multicomponente de 2-(2-piridil)quinolinas via reação de Povarov

no anel piridina, nota-se que a atividade é dependente da natureza dos substituintes, bem como o padrão de substituição dos mesmos, quando se considera o anel quinolina. Dentre os compostos 13,15 e 22, que contém o anel quinolina dissubstituído com grupos metoxila em posições para, orto e meta, respectivamente, observou-se que 22 foi o mais ativo, sendo a atividade superior à do miconazol (C. albicans) e à da nistatina (C. krusei). Para o composto 16, que contém o grupo metilenodioxi, a atividade variou de baixa a moderada contra todos os micro-organismos ensaiados.

De acordo com os resultados obtidos, o mais ativo e promissor antifúngico é o composto $\mathbf{2 0}$, que contém o anel quinolina não substituído. Sua atividade foi superior à do miconazol contra $C$. albicans, $C$. glabrata, C. tropicalis e C. neoformans, e superior à da nistatina contra $C$. kruseis e C. neoformans. As porcentagens de inibição causadas pelo composto 22, com o anel quinolina dissubstituído, também pode ser equiparada àquela dos antifúngicos comerciais. Esses resultados são bastante relevantes, considerando que atualmente a maioria dos casos relatados de infecções nosocomiais tem sido atribuída a $C$. tropicalis, C. glabrata, C. parapsilosis, C. krusei e outras espécies de Candida spp. não-albicans (Kouznetsov et al., 2012). ${ }^{10}$ Ressaltase a diferente seletividade observada com os compostos 20 e 22 em relação à nistatina. A porcentagem de inibição sobre $C$. neoformans foi superior à do antifúngico padrão no caso do composto 20, enquanto aquela causada pelo composto $\mathbf{2 2}$ foi significativamente menor.

Para os compostos promissores 6, 8, 12, 15-17 e 19-25 foram determinados os valores de $\mathrm{IC}_{50}$, e comparados com os controles miconazol e nistatina conforme apresentado na Tabela 4.

Como pode ser verificado na Tabela 4 , não foi possível calcular os valores de $\mathrm{IC}_{50}$ para todos os micro-organismos, uma vez que em diversos casos não se observou uma boa correlação entre atividade-concentração.

No caso de C. albicans, todos os compostos foram menos ativos que os produtos comerciais. O composto mais ativo foi o $\mathbf{2 0}$, com $\mathrm{IC}_{50}=37,9 \mu \mathrm{g} \mathrm{mL}^{-1}$, correspondendo a 4,8 vezes o valor encontrado para a nistatina. No caso do composto $\mathbf{2 2}$, que na concentração de $250 \mu \mathrm{g} \mathrm{mL}^{-1}$ apresentou atividade semelhante aos antifúngicos comerciais causando $96,4 \%$ de inibição, observou-se um valor de $\mathrm{IC}_{50}$ muito elevado. 
Tabela 3. Porcentagens de inibição do crescimento de cinco espécies de Candida spp. e C. neoformans causado por uma série de 2-(2-piridil)quinolinas na concentração de $250 \mu \mathrm{g} \mathrm{mL}^{-1}$

\begin{tabular}{|c|c|c|c|c|c|c|}
\hline Composto & $\mathrm{Ca}^{\mathrm{a}}$ & $\mathrm{Cg}^{\mathrm{a}}$ & $\mathrm{Ct}^{\mathrm{a}}$ & $\mathrm{Cp}^{\mathrm{a}}$ & $\mathrm{Ck}^{\mathrm{a}}$ & $\mathrm{Cn}^{\mathrm{a}}$ \\
\hline 5 & $3,1 \pm 0,1$ & $22,6 \pm 0,4$ & $16,9 \pm 0,3$ & $6,1 \pm 5,4$ & $10,8 \pm 0,2$ & 0 \\
\hline 6 & $32,9 \pm 2,4$ & $61,6 \pm 0,6$ & $53,3 \pm 4,4$ & $48,9 \pm 0,5$ & $57,0 \pm 0,1$ & $4,7 \pm 2,0$ \\
\hline 7 & $16,3 \pm 1,5$ & $26,0 \pm 1,1$ & $41,3 \pm 0,7$ & $32,82 \pm 0,74$ & $31,56 \pm 0,63$ & $48,86 \pm 0,79$ \\
\hline 8 & $56,8 \pm 3,1$ & $44,1 \pm 0,6$ & $32,4 \pm 2,4$ & $26,6 \pm 3,1$ & $30,9 \pm 0,6$ & $41,4 \pm 0,8$ \\
\hline 12 & $37,6 \pm 0,2$ & $59,1 \pm 4,2$ & $56,2 \pm 0,9$ & $38,1 \pm 0,1$ & $46,2 \pm 2,8$ & $65,0 \pm 0,4$ \\
\hline 13 & $7,9 \pm 3,9$ & $23,0 \pm 4,3$ & $24,3 \pm 3,6$ & $24,5 \pm 3,9$ & $5,6 \pm 3,1$ & $42,9 \pm 2,4$ \\
\hline 14 & $13,8 \pm 0,6$ & $30,0 \pm 2,7$ & $34,1 \pm 1,0$ & $25,1 \pm 2,0$ & $22,1 \pm 0,2$ & 0 \\
\hline 15 & $11,3 \pm 0,8$ & 0 & $33,9 \pm 0,6$ & $6,6 \pm 3,4$ & $23,1 \pm 1,3$ & $61,9 \pm 3,2$ \\
\hline 16 & $41,3 \pm 1,8$ & $58,6 \pm 1,4$ & $73,9 \pm 0,1$ & $59,8 \pm 3,7$ & $40,3 \pm 4,1$ & $58,6 \pm 1,2$ \\
\hline 17 & $6,4 \pm 0,7$ & $74,5 \pm 1,1$ & $51,6 \pm 2,0$ & $10,9 \pm 1,1$ & $38,1 \pm 1,7$ & $53,6 \pm 2,0$ \\
\hline 18 & $27,0 \pm 3,5$ & $34,9 \pm 0,4$ & $32,3 \pm 2,5$ & $5,9 \pm 1,0$ & $33,4 \pm 0,9$ & $19,1 \pm 2,8$ \\
\hline 19 & $20,0 \pm 3,2$ & $44,1 \pm 2,9$ & $54,6 \pm 0,8$ & $35,0 \pm 1,0$ & $25,3 \pm 4,2$ & $60,0 \pm 0,4$ \\
\hline 20 & $97,9 \pm 0,5$ & $96,7 \pm 0,9$ & $95,6 \pm 1,5$ & $95,8 \pm 0,3$ & $96,1 \pm 0,2$ & $85,5 \pm 0,6$ \\
\hline 21 & 0 & 0 & $41,3 \pm 4,4$ & $8,8 \pm 2,5$ & $60,8 \pm 3,2$ & 0 \\
\hline 22 & $96,4 \pm 0,1$ & $90,4 \pm 1,3$ & $93,7 \pm 0,7$ & $87,1 \pm 1,5$ & $96,3 \pm 0,3$ & $53,7 \pm 0,7$ \\
\hline 23 & $3,3 \pm 12,5$ & 0 & $39,2 \pm 10,9$ & 0 & $51,8 \pm 6,3$ & 0 \\
\hline 24 & $30,4 \pm 3,1$ & $83,2 \pm 8,8$ & $74,5 \pm 6,8$ & $76,1 \pm 6,1$ & $78,2 \pm 4,8$ & $25,5 \pm 1,5$ \\
\hline 25 & $14,6 \pm 1,7$ & $24,6 \pm 4,3$ & $53,3 \pm 0,9$ & $28,9 \pm 11,7$ & $72,5 \pm 2,9$ & 0 \\
\hline 26 & $18,6 \pm 1,0$ & $11,3 \pm 1,8$ & $7,7 \pm 5,4$ & $1,9 \pm 2,0$ & 0 & $14,7 \pm 1,6$ \\
\hline Miconazol & 94,0 & 96,2 & 99,8 & 96,1 & 99,3 & 71,9 \\
\hline Nistatina & 98,2 & 98,3 & 98,4 & 96,8 & 95,2 & 83,3 \\
\hline
\end{tabular}

${ }^{a}$ Ca: Candida albicans; $\mathrm{Cg}$ : C. glabrata; Ct: Candida tropicalis; Cp: Candida parapsilosis; Ck: Candida krusei; Cn: Cryptococcus neoformans.

Tabela 4. Concentração Inibitória $\mathrm{IC}_{50}\left(\mu \mathrm{g} \mathrm{mL}^{-1}\right)$ de 2-(2-piridil)quinolinas

\begin{tabular}{ccccccc}
\hline Composto & $\mathrm{Ca}^{\mathrm{a}}$ & $\mathrm{Cg}^{\mathrm{a}}$ & $\mathrm{Cp}^{\mathrm{a}}$ & $\mathrm{Cn}^{\mathrm{a}}$ & $\mathrm{Ct}^{\mathrm{a}}$ & $\mathrm{Ck}^{\mathrm{a}}$ \\
\hline $\mathbf{6}$ & $-{ }^{\mathrm{b}}$ & 157,7 & - & - & - & - \\
$\mathbf{8}$ & 149,9 & - & - & - & - & - \\
$\mathbf{1 2}$ & 131,8 & - & - & 111,7 & 179,8 & - \\
$\mathbf{1 5}$ & - & - & - & 48,3 & - & - \\
$\mathbf{1 6}$ & 135,4 & - & - & 96,9 & - & - \\
$\mathbf{1 7}$ & 152,2 & - & - & - & 231,9 & - \\
$\mathbf{1 9}$ & - & - & - & 125,2 & 158,1 & - \\
$\mathbf{2 0}$ & 37,9 & 36,1 & 62,8 & 20,8 & 49,8 & $<1,95$ \\
$\mathbf{2 1}$ & - & - & - & - & - & $<1,95$ \\
$\mathbf{2 2}$ & 126,5 & 133,9 & 178,9 & 224,8 & $<1,95$ & $<1,95$ \\
$\mathbf{2 3}$ & - & - & - & - & - & $<1,95$ \\
$\mathbf{2 4}$ & 66,2 & - & - & - & 4,6 & $<1,95$ \\
$\mathbf{2 5}$ & - & - & - & - & 215 & $<1,95$ \\
Miconazol & 2,4 & 8,7 & 1,9 & 1,9 & 1,9 & 2,2 \\
Nistatina & 7,8 & 10,6 & 19,6 & 7,9 & 10 & 12,4 \\
\hline
\end{tabular}

${ }^{a}$ Ca: Candida albicans, $\mathrm{Cg}$ : Candida glabrata, $\mathrm{Ct}:$ Candida tropicalis, Cp: Candida parapsilosis, Ck: Candida krusei, Cn: Cryptococcus neoformans. b -: $\mathrm{IC}_{50}$ não foi determinado.

Para C. glabrata, foram observados resultados semelhantes aos encontrados para C. albicans, onde o composto 20 também se mostrou mais ativo. Nesse caso o valor de $\mathrm{IC}_{50}(36,1 \mu \mathrm{g} \mathrm{mL}-1)$ corresponde a 4,1 e 3,4 vezes o valor encontrado para os produtos miconazol e nistatina, respectivamente. O segundo composto mais ativo foi também o 22, com aproximadamente o mesmo valor de $\mathrm{IC}_{50}$, bem acima de $100 \mu \mathrm{g} \mathrm{mL}-1 . \mathrm{O}$ mesmo comportamento foi encontrado para $C$. parapsilosis e $C$. neoformans, em que se verificou que dentre todos os compostos, $\mathbf{2 0}$ foi o mais ativo, embora ainda bem menos potente que os produtos comerciais.

Para a espécie C. parapsilosis não foi possível calcular os valores de $\mathrm{IC}_{50}$ para a maioria dos compostos, à exceção dos compostos 20
$\left(\mathrm{IC}_{50}=62,8 \mu \mathrm{g} \mathrm{mL} \mathrm{L}^{-1}\right)$ e $\mathbf{2 2}\left(\mathrm{IC}_{50}=178,9 \mu \mathrm{g} \mathrm{mL}-1\right)$. Em ambos os casos, as atividades observadas foram menores que aquelas dos produtos comerciais. Esses resultados são consistentes com os observados na Tabela 3, na qual se verifica que esses dois compostos foram os únicos que causaram inibição acima de $90 \%$ para esses micro-organismos, quando testados na concentração de $250 \mu \mathrm{g} \mathrm{mL}^{-1}$.

Para C. kruzei foi verificado que seis compostos (20-25) foram mais ativos $\left(\mathrm{IC}_{50}<1,95 \mu \mathrm{g} \mathrm{mL}-1\right)$ que os produtos comerciais.

Em termos de seletividade dos compostos em relação aos diversos micro-organismos, verificou-se que o composto 15 é seletivo para a levedura $C$. neoformans $\left(\mathrm{IC}_{50}=48,3 \mu \mathrm{g} \mathrm{mL} \mathrm{m}^{-1}\right)$, porém, o valor de $\mathrm{IC}_{50}$ é moderado sendo 25,4 e 6,1 vezes maior em comparação ao observado para o miconazol e nistatina, respectivamente. $\mathrm{O}$ composto $\mathbf{2 0}$, embora tenha apresentado valores de $\mathrm{IC}_{50}$ na faixa de $<1,95-49,8 \mu \mathrm{g}$ $\mathrm{mL}^{-1}$, apresentou certa seletividade em relação a C. kruzei.

Por sua vez, o composto $\mathbf{2 2}$ foi seletivo e muito ativo contra a C. tropicalis $\left(\mathrm{IC}_{50}<1,95 \mu \mathrm{g} \mathrm{mL}-1\right)$ e C. krusei $\left(\mathrm{IC}_{50}<1,95 \mu \mathrm{g} \mathrm{mL}^{-1}\right)$. Resultado similar foi observado para o composto 24. Finalmente os compostos 23 e $\mathbf{2 5}$ somente foram muito ativos contra C. krusei $\left(\mathrm{IC}_{50}<1,95 \mu \mathrm{g} \mathrm{mL}^{-1}\right)$.

Considerando que a diferença estrutural entre os compostos é bastante limitada, não é possível tirar uma conclusão minuciosa sobre a relação estrutura-atividade. Entretanto, pelos resultados dos ensaios biológicos, fica evidente que a maioria dos compostos contendo grupo metoxi ou metilenodioxi no anel quinolínico são pouco ativos ou inativos. Apesar disso, o composto 22, que apresenta grupos metoxi nos carbonos $\mathrm{C} 6$ e $\mathrm{C} 8$, é muito ativo contra dois dos micro-organismos testados. Isso indica que a natureza do grupo e a sua posição são relevantes para a atividade biológica.

Os compostos 16 e 23 diferem apenas pela presença de um átomo de bromo no anel piridina. Pelos dados da Tabela 3, verifica-se que na concentração de $250 \mu \mathrm{g} \mathrm{mL}^{-1} \mathrm{o}$ composto $\mathbf{1 6}$ apresentou em média atividade maior sobre a maioria dos micro-organismos, enquanto $\mathbf{2 3}$ causou mais de $50 \%$ de inibição apenas contra C. kruzei. Entretanto, 
ao se avaliar os dados da Tabela 4 , os valores de $\mathrm{IC}_{50}$ para 16 puderam ser calculados apenas para dois micro-organismos e foram muito elevados. No caso de $\mathbf{2 3}$ (composto bromado), o valor de $\mathrm{IC}_{50}$ foi calculado apenas para $C$. kruzei e foi muito baixo. Comparando também os efeitos dos compostos $\mathbf{2 4}$ e $\mathbf{2 5}$, que diferem apenas por um átomo de bromo no anel piridínico em $\mathbf{2 5}$, verifica-se grande variação nos efeitos sobre os vários micro-organismos.

Assim, os dados indicam que os efeitos dos compostos sobre os micro-organismos podem ser modulados pela alteração dos grupos, e suas posições, nas duas unidades estruturais.

\section{CONCLUSÃO}

Foi desenvolvido um procedimento eficiente para a síntese de uma série de 2-(2-piridil)quinolinas empregando a reação multicomponentes de Povarov sob irradiação por micro-ondas. Em comparação com o aquecimento convencional, observou-se que o emprego de micro-ondas resultou, em alguns casos, na melhora nos rendimentos, bem como permitiu a síntese de compostos não acessíveis por aquecimento convencional. O emprego de micro-ondas resultou ainda em uma considerável redução no tempo reacional, passando de $24 \mathrm{~h}$ para $2 \mathrm{~h}$.

Todos os compostos obtidos causaram algum nível de inibição do crescimento dos fungos selecionados. As quinolinas $\mathbf{2 0}$ e $\mathbf{2 2}$ foram as mais ativas e mostraram amplo espectro de ação antifúngica, especialmente contra Candida tropicalis e C. neoformans. A espécie $C$. krusei mostrou-se mais sensível frente a seis quinolinas $\left(\mathrm{IC}_{50}<1,95 \mu \mathrm{g} \mathrm{mL} \mathrm{L}^{-1}\right.$ para os compostos 20-25).

Considerando a eficiência e simplicidade da metodologia desenvolvida, e o amplo espectro de atividades dos compostos obtidos contra uma série de fungos de importância médica, antecipamos que esse trabalho possa ser aplicado na obtenção de compostos com maior diversidade estrutural e mais ativos.

\section{MATERIAL SUPLEMENTAR}

Material suplementar está disponível em http://quimicanova.sbq. org.br, na forma de arquivo PDF, com acesso livre.

\section{AGRADECIMENTOS}

Ao CNPq e a FAPEMIG pelas bolsas concedidas e pelo apoio financeiro. Aos técnicos Cristiane Cerceau pela obtenção dos espectros de RMN e José Luiz Pereira pelos espectros de massas.

\section{REFERÊNCIAS}

1. Kathiravan, M.; Salake, A. B.; Chothe, A.; Dudhe, P. B.; Watode, R. P.; Mukta, M. S.; Gadwe, S.; Bioorg. Med. Chem. 2012, $20,5678$.

2. Vicente, M. F.; Basílio, A.; Cabello, A.; Peláez, F.; Clin. Microbiol. Infect. 2003, 9, 15 .

3. Ostrosky-Zeichner; L.; Am. J. Med. 2012, 125, 14.

4. Scorzoni, L.; Sangalli-Leite, F.; Singulani, J. L.; Silva, A. C. A. P.; Costa-Orlandi, C. B.; Fusco-Almeida, A. M.; Mendes-Giannini, M. J. S.; J. Microbiol. Methods 2016, 123, 68.

5. Pfaller, M. A.; Diekema, D. J.; Clin. Microbiol. Rev. 2007, 20, 133.

6. Wilson, L. S.; Reyes, C. M.; Stolpman, M.; Speckman, J.; Allen, K.; Beney, J.; Value Health 2002, 5, 26.

7. Lam, P. -L.; Lee, K. K. -H.; Kok, S. H. -L.; Gambari, R.; Lam, K. -H.; Ho, C. -L.; Ma, X.; Lo, Y. -L.; Wong, W. -Y.; Dong, Q. -C.; Bian, Z. -X.; Chui, C. -H.; RSC Adv. 2016, 6, 104575.

8. Garibotto, F. M.; Garro, A. D.; Masman, M. F.; Rodriguéz, A. M.; Luiten, P. G. M.; Raimond, M.; Zacchino, S. A.; Somlai, C.; Penke, B.; Enriz, R. D.; Bioorg. Med. Chem. 2010, 18, 158.
9. Hadrich, I.; Makni, F.; Neji, S.; Abbes, S.; Cheikhrouhou, F.; Trabelsi, H.; Sellami, H.; Ayadi, A.; Mycopathologia 2012, 174, 131.

10. Kouznetsov, V. V.; Gómez, C. M. M.; Derita, M. G.; Svetaz, L.; Olmo, E.; Zacchino, S. A.; Bioorg. Med. Chem. 2012, 20, 6506.

11. Romano, G.; Costantini, M.; Sansone, C.; Lauritano, C.; Ruocco, N.; Ianora, A. Mar. Environ. Res. 2016, 1.

12. Moreira, M. D.; Picanço, M. C.; Barbosa, L. C. A.; Guedes, R. N. C.; Campos, M. R.; Silva, G. A.; Martins, J. C.; Pesqui. Agropec. Bras. 2007, 42, 909 .

13. Santos, E. T.; Pereira, M. L. A.; Silva, C. F. P. G.; Souza-Neta, L. C.; Geris, R.; Martins, D.; Santana, A. E. G.; Barbosa, L. C. A.; Silva, H. G. O.; Freitas, G. C.; Figueiredo, M. P.; Oliveira, F. F.; Batista, R.; Int. J. Mol. Sci. 2013, 14, 8496.

14. Bracarense, A. A. P.; Takahashi, J. A.; Braz. J. Microbiol. 2014, 45, 313.

15. Rajeshkumar, R.; Sundararaman, M.; Mycoses 2012, 55, 60.

16. Varejão, E. V. V.; Demuner, A. J.; Barbosa, L. C. A.; Barreto, W. B.; Crop. Prot. 2013, 48, 41.

17. Blackie, M. A. L.; Beagley, P.; Croft, S. L.; Hendrick, H.; Moss, J. R.; Chibale, K.; Bioorg. Med. Chem. 2007, 15, 6510.

18. Makawana, J. A.; Sangani, C. B.; Teraiya, S. B.; Zhu, H. L.; Med. Chem. Res. 2014, 23, 471.

19. Chen, M.; Chen, H.; Ma. J. W.; Liu, X. Y.; Zhang, S. Y.; Bioorg. Med. Chem. Lett. 2014, 24, 2876.

20. Chen, Y.; Zhao, Y.; Lu, C.; Tzeng, C.; Wang, J.; Bioorg. Med. Chem. 2006, 14, 4373 .

21. Pereira, G. R.; Brandão, L. M.; Arantes, H. A.; Paula, R. C.; Nascimento, M. F. A.; Santos, F. M.; Rocha, R. K.; Lopes, J. C. D.; Oliveira, A. B.; J. Med. Chem. 2014, 73, 295.

22. Musiol, R.; Curr. Pharm. Des. 2013, 19, 1835.

23. Rao, K. V.; Cullen, W. P.; Antibiot. Аnnu. 1959, 7, 950.

24. Xu, F.; Kong, D.; He, X.; Zhang, Z.; Han, M.; Xie, X.; Wang, P.; Cheng, H.; Tao, M.; Zhang, L.; Deng, Z.; Lin, S. J.; J. Am. Chem. Soc. 2013, 135,1739

25. Xia, X.; Lin, S.; Xia, X. X.; Comg, F. S.; Zhong, J. J.; J. Appl. Microbiol. Biotechnol. 2014, 98, 4399.

26. Cai, W.; Hassani, M.; Karki, R.; Walter, E. D.; Koelsch, K. H.; Seradj, H.; Lineswala, J.; Mirzaei, H.; York, J. S.; Olang, F.; Sedighi, M.; Lucas, J. S.; Eads, T. J.; Rose, A. S.; Charkhzarrin, S.; Hermann, N. G.; Beall, H. D.; Behforouz, M.; Bioorg. Med. Chem. 2010, 18, 1899.

27. Bringmann, G.; Reichert, Y.; Kane, V. V.; Tetrahedron 2004, 60, 3539

28. Donohoe, J. T.; Jones, C.; Barbosa, L. C. A.; J. Am. Chem. Soc. 2011, $133,16418$.

29. Donohoe, J. T.; Christopher, R. J.; Kornahrens, A. F.; Barbosa, L. C. A.; Walport, L. J.; Tatton, M. R.; O’Hagan, M.; Rathi, A. H.; Baker, D. B.; J. Org. Chem. 2013, 78, 12338.

30. Vandekerckhove, S.; Tran, H. G.; Desmet, T.; D'hooghe, M.; Bioorg. Med. Chem. Lett. 2013, 23, 4641.

31. Borel, C. R.; Barbosa, L. C. A.; Maltha, C. R. A.; Fernandes, S. A.; Tetrahedron Lett. 2015, 56, 662.

32. Pereira, U. A.; Barbosa, L. C. A.; Demuner, A. J.; Silva, A. A.; Bertazzini, M.; Forlani, G.; Chem. Biodivers. 2015, 12, 987.

33. Pereira, U. A.; Moreira, T. A.; Barbosa, L. C. A.; Maltha, C. R. A.; Bomfim, I. S.; Maranhão, S. S.; Moraes, M. O.; Pessoa, C.; BarrosNepomuceno, F. W. A.; Med. Chem. Commun. 2016, 7, 345.

34. Rodríguez-Hernandez, D.; Demuner, A. J.; Barbosa, L. C. A.; Heller, L.; Csuk, R.; Eur. J. Med. Chem. 2016, 115, 257.

35. Perrin, D. D.; Armarego, W. L. F.; Purification of Laboratory Chemicals, $3^{\text {rd }}$ ed., Pergamon: Oxford, 1988.

36. Clinical and Laboratory Standards Institute; Reference Method for Broth Dilution Antifungal Susceptibility Testing of Yeasts, $3^{\text {rd }}$ ed., M27-A2.V. 22, n 15, 2002.

37. Barbosa, L. C. A. Espectroscopia no Infravermelho na caraterização de compostos orgânicos, $1^{\mathrm{a}}$ ed., Editora UFV: Viçosa, 2011. 